\title{
HOW TO INCORPORATE THE SPRING-MASS CONDITIONS IN FINITE-DIFFERENCE SCHEMES.
}

\author{
BRUNO LOMBARD* AND JOËL PIRAUX ${ }^{\dagger}$
}

\begin{abstract}
The spring-mass conditions are an efficient way to model imperfect contacts between elastic media. These conditions link together the limit values of the elastic stress and of the elastic displacement on both sides of interfaces. To insert these spring-mass conditions in classical finitedifference schemes, we use an interface method, the Explicit Simplified Interface Method (ESIM). This insertion is automatic for a wide class of schemes. The interfaces do not need to coincide with the uniform cartesian grid. The local truncation error analysis and numerical experiments show that the ESIM maintains, with interfaces, properties of the schemes in homogeneous medium.
\end{abstract}

Key words. elastic waves, interface methods, jump conditions, discontinuous coefficients, imperfect contact, hyperbolic conservation laws.

AMS subject classifications. 35L40, 65M06

1. Introduction. Let us consider a one-dimensional elastic medium with one interface at $x=\alpha$, as shown in Fig. 1.1. To study the wave propagation in this medium, we must define the conditions that are satisfied by the elastic stress and by the elastic displacement on both sides of the interface. Classically, the perfect conditions are used at $x=\alpha$ : they impose respectively the continuity of the elastic stress and of the elastic displacement across the interface [4]. The perfect conditions idealize bonded contacts between solids; then, the interface is called a perfect interface.

Because of defects, like air or cracks, the contacts between solids are often not perfect, and a jump of the elastic stress and of the elastic displacement can occur across $\alpha$. Authors from various disciplines, like the non-destructive evaluation of materials or the geophysics, have modeled such situations by the spring-mass conditions. These conditions satisfied by wave fields across $\alpha$ are analogous to mechanical laws of springs [1], [21], [22], [26] or springs and masses [2], [25]. Stiffness and mass values are expected to be connected, although not necessarily in a trivial way, to the contact quality [7], [28]. Then, the interface is called an imperfect interface.

Even if the spring-mass conditions are extensively used in physics to model realistic situations, they are rarely investigated for numerical simulations in the time domain. To our knowledge, the first attempt is done by Punjani and Bond [24]. Coates and Schoenberg incorporate these conditions in a staggered-grid scheme using an equivalent medium theory [5]. Gu, Nihei and Myer follow a boundary integral approach [11], [12]. Delsanto and Scalerandi investigate the spring-mass conditions in the framewok of the local interaction simulation approach (LISA) [7]. Despite their qualities, all these approaches have three classes of limitations. Firstly, they are dedicated to one given scheme. Secondly, the inertial effects are not taken into account, although they can be preponderant [25]. Thirdly, no attempt is made to control the numerical accuracy near the interfaces. The purpose of this article is to overcome these drawbacks: we propose a procedure to incorporate the spring-mass conditions in a wide class of classical finite-difference schemes. Moreover, the numerical properties of the resulting schemes, like the local truncation error, are maintained near the

\footnotetext{
*Laboratoire de Mécanique et d'Acoustique, 31 chemin Joseph Aiguier, 13402 Marseille, France(lombard@lma.cnrs-mrs.fr).

${ }^{\dagger}$ Laboratoire de Mécanique et d'Acoustique, 31 chemin Joseph Aiguier, 13402 Marseille, France(piraux@lma.cnrs-mrs.fr).
} 
interfaces.

To do so, we use the Explicit Simplified Interface Method (ESIM) [23] previously developed in the simple case of fluid/fluid perfect interfaces; the goal in [23] was only to maintain the order of accuracy with discontinuous coefficients. This method is an extension of the Immersed Interface Method (IIM) [17], [18], [30], [32], [33] developed by LeVeque and his collaborators, and of the Explicit Jump Immersed Interface Method (EJIIM) [29], [31] developed by Wiegmann and Bube. The ESIM is preferred for two reasons: firstly, it is coupled with any scheme automatically, and secondly, it maintains properties that have the schemes in homogeneous medium.

The technique proposed in this paper is very simple. The interfaces are "immerged" in the regular cartesian grid: we do not need to raffine the meshing to make grid points coincide with interfaces. This greatly simplifies 2D-3D computations with arbitrary-shaped interfaces. At grid points near interfaces, the chosen scheme is modified implicitly through an explicit modification of the numerical values used for time-stepping. This modification is based on the spring-mass conditions satisfied at $\alpha$ by the exact solution. The additional computational cost induced by the ESIM is negligible.

The paper is organized as follows. In 2, we recall the one-dimensional elastodynamics equations and some explicit numerical schemes. The spring-mass conditions are proposed in 3. The interface method is explained in 4. Numerical experiments in 5 show comparisons with analytical solutions and some measures of convergence on realistic configurations. This paper is devoted to one-dimensional algorithms: however, we conclude our presentation with a two-dimensional simulation, showing the efficiency of the method.

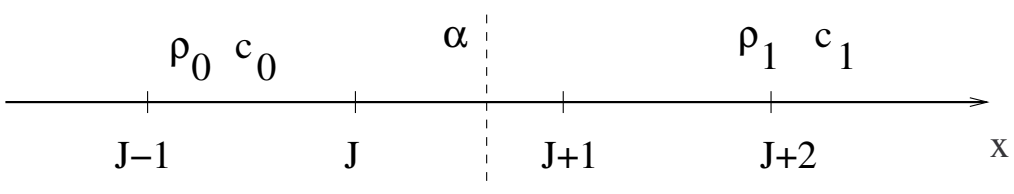

FIG. 1.1. 1D elastic medium with one imperfect interface

\section{Wave propagation and numerical schemes.}

2.1. Conservation laws. For the sake of simplicity, the physical parameters are supposed piecewise constant around the interface at $x=\alpha$, as shown in Fig. 1.1, with

$$
(\rho, c)=\left\{\begin{array}{lll}
\left(\rho_{0}, c_{0}\right) & \text { if } & x \leq \alpha \\
\left(\rho_{1}, c_{1}\right) & \text { if } & x>\alpha,
\end{array}\right.
$$

where $\rho$ and $c$ are respectively the density and the celerity of elastic waves. Outside $\alpha$, the one-dimensional elastodynamics equations can be written as a first-order linear hyperbolic system

$$
\begin{gathered}
\rho \frac{\partial v}{\partial t}=\frac{\partial \sigma}{\partial x} \\
\rho c^{2} \frac{\partial v}{\partial x}=\frac{\partial \sigma}{\partial t},
\end{gathered}
$$


where $v(x, t)$ and $\sigma(x, t)$ are respectively the elastic velocity and the elastic stress. We set

$$
\boldsymbol{U}=\left(\begin{array}{c}
v \\
\sigma
\end{array}\right), \quad \boldsymbol{A}=\left(\begin{array}{cc}
0 & -\frac{1}{\rho} \\
-\rho c^{2} & 0
\end{array}\right) \text {. }
$$

So, we consider the initial value problem

$$
\begin{aligned}
& \frac{\partial}{\partial t} \boldsymbol{U}+\boldsymbol{A} \frac{\partial}{\partial x} \boldsymbol{U}=\mathbf{0} \quad \text { for } x \in \mathbb{R}, x \neq \alpha, t \geq t_{0}, \\
& \boldsymbol{U}\left(x, t_{0}\right)=\boldsymbol{U}_{0}(x) \quad \text { for } x \in \mathbb{R},
\end{aligned}
$$

where $\boldsymbol{U}_{0}$ is a sufficiently smooth $C^{m}$ function. We easily deduce from (2.3) and from (2.4) higher order relations between spatial and time derivatives, sumed up as follows.

RESUlT 1. Time derivatives and spatial derivatives of $\boldsymbol{U}(x, t)$ satisfy

$$
\begin{gathered}
\frac{\partial^{2 k}}{\partial t^{2 k}} \boldsymbol{U}=c^{2 k} \frac{\partial^{2 k}}{\partial x^{2 k}} \boldsymbol{U} \\
\frac{\partial^{2 k+1}}{\partial t^{2 k+1}} \boldsymbol{U}=-c^{2 k} \boldsymbol{A} \frac{\partial^{2 k+1}}{\partial x^{2 k+1}} \boldsymbol{U} .
\end{gathered}
$$

Throughout this paper, we designate the matrix $\boldsymbol{A}$ by $\boldsymbol{A}_{0}$ if $x \leq \alpha$, or by $\boldsymbol{A}_{1}$ if $x>\alpha$.

2.2. Numerical schemes. To integrate (2.4) in homogeneous medium, we introduce a uniform grid of points $\left(x_{i}, t_{n}\right)=(i \Delta x, n \Delta t)$, where $\Delta x$ is the mesh size and $\Delta t$ is the time step. Then, we seek an approximation $\boldsymbol{U}_{i}^{n}$ of $\boldsymbol{U}\left(x_{i}, t_{n}\right)$. To do so, we use two-step, explicit, and $(2 s+1)$-point spatially-centered finite-difference schemes, where $s$ is the width of the stencil of the scheme. Time-stepping of two-stage schemes can be written symbolically

$$
\boldsymbol{U}_{i}^{n+1}=\boldsymbol{U}_{i}^{n}+\boldsymbol{H}\left(\boldsymbol{U}_{i-s}^{n}, \ldots, \boldsymbol{U}_{i+s}^{n}\right),
$$

where the discrete operator $\boldsymbol{H}: \mathbb{R}^{2 \times(2 s+1)} \rightarrow \mathbb{R}^{2}$ is continuous [10]. For numerical experiments performed in 5 , we use three schemes of increasing complexity and quality: the Lax-Wendroff scheme, a finite volume scheme with flux limiter, and a WENO 5 scheme. These three schemes satisfy the Courant-Friedrichs-Lewy (CFL) condition of stability in homogeneous medium, that is

$$
\mathrm{CFL}=\max \left(c_{0}, c_{1}\right) \frac{\Delta t}{\Delta x} \leq 1 .
$$

These schemes, issued from computational fluid mechanics, are classical: so, we do not detail their implementation. We only refer to surveys articles, and we sketch their main properties. The Lax-Wendroff scheme $(s=1)$ is written

$$
\boldsymbol{U}_{i}^{n+1}=\boldsymbol{U}_{i}^{n}-\frac{\Delta t}{2 \Delta x} \boldsymbol{A}\left(\boldsymbol{U}_{i+1}^{n}-\boldsymbol{U}_{i-1}^{n}\right)+\frac{1}{2}\left(\frac{c \Delta t}{\Delta x}\right)^{2}\left(\boldsymbol{U}_{i+1}^{n}-2 \boldsymbol{U}_{i}^{n}+\boldsymbol{U}_{i-1}^{n}\right) .
$$

The Lax-Wendroff scheme is second-order accurate both in space and time; it is easy to implement, and its computational cost is very low [15]. However, it introduces 
numerical dispersion that damages the numerical solution. To avoid the spurious oscillations induced by the numerical dispersion, one can use second-order finite volume schemes described in [14]. These schemes $(s=2)$ introduce locally numerical diffusion via nonlinear flux limiters. This diffusion leads to a very precise spatial localization of waves, but it flattens their crests and damages their amplitude. Lastly, WENO schemes control the numerical dispersion without damaging the amplitudes of waves, but they are more expensive on a computational point of view. Here, we use a WENO 5 scheme described in [13], which is fifth-order accurate in space and fourth-order accurate in time. To be rigorous, its time-marching is not (2.6), since the WENO schemes lie on Runge-Kutta integrations between $t_{n}$ and $t_{n+1}$.

To conclude this section, we make two remarks. Firstly, the choice of the scheme is not essential for the method proposed further: one could also choose to integrate the scalar elastic wave equation with classical, multistep, and explicit finite-difference schemes [27]: the further discussion remains the same with only minor changes. Secondly, as shown in Fig. 1.1, we define an integer $J$ by

$$
x_{J} \leq \alpha<x_{J+1}
$$

If a grid point coincides with the interface, the convention (2.9) about $J$ avoids any ambiguity. A grid point is irregular if its time-stepping crosses the interface; otherwise, a grid point is regular. According to the width $s$ of the chosen scheme (2.6), irregular points are

$$
x_{J-s+1}, \ldots, x_{J+s} \text {. }
$$

The time-stepping (2.6) is only applied at the regular points. The time-stepping at irregular points is detailed and analysed in section 4 .

3. The spring-mass conditions. Classically, only perfect conditions are considered at interfaces. Writing for any function $f(x, t)$

$$
\left[f(\alpha, t]=\lim _{x \rightarrow \alpha^{+}} f(x, t)-\lim _{x \rightarrow \alpha^{-}} f(x, t),\right.
$$

these perfect conditions are

$$
[u(\alpha, t)]=0, \quad[\sigma(\alpha, t)]=0,
$$

where $u(x, t)$ is the elastic displacement and $\sigma(x, t)$ is the elastic stress. The perfect conditions (3.1) idealize a perfect contact at $\alpha$ between solids.

To describe an imperfect contact at $\alpha$, one can generalize (3.1) via the springmass conditions. Given two constants $K>0, M \geq 0$, respectively called the stiffness and the mass of the interface, the spring-mass conditions are

$$
\begin{aligned}
& {\left[u(\alpha, t]=\frac{1}{K} \sigma\left(\alpha^{-}, t\right),\right.} \\
& {[\sigma(\alpha, t)]=M \frac{\partial^{2}}{\partial t^{2}} u\left(\alpha^{-}, t\right) .}
\end{aligned}
$$

The conditions (3.2) are called the spring-mass conditions because of analogies with the equations governing a spring-mass system. Authors have found that they are a good model for imperfect contacts like fractures [26]. 
The derivation of (3.2) in the case of a thin intermediate layer between media 0 and 1 is proposed in Appendix A; then, $K$ and $M$ are deduced from the parameters of the intermediate layer (A.4). This academic case is of interest for modeling wave propagation across a glue layer. Some authors have also derived the spring-mass conditions from an asymptotic analysis of diffraction of elastic waves on cracks [1].

The spring-mass conditions are an easy way to take into account all degrees of contact between solids, from the perfect contact to free extremities. Indeed, the perfect conditions (3.1) are recovered if $K=+\infty$ and $M=0$. If $K \rightarrow 0$ and $M=0$, the spring-mass conditions (3.2) induce $\sigma\left(\alpha^{-}, t\right)=\sigma\left(\alpha^{+}, t\right)=0$, hence both sides of $\alpha$ are stress-free extremities.

In the next two paragraphs, we calculate the conditions that are satisfied by $\boldsymbol{U}(x, t)$ and by its spatial derivatives across the imperfect interface. These conditions are required by the numerical method exposed in 4 . In the general case $M \neq 0$ or $K \neq+\infty$, the spring-mass conditions (3.2) are not the same if $\alpha^{+}$and $\alpha^{-}$are exchanged. The limit values of $\boldsymbol{U}(x, t)$ and of its spatial derivatives therefore will be examined successively on both sides of $\alpha$.

3.1. Jump of the solution. We differentiate the first equation of (3.2) with respect to $t$; since $v=\frac{\partial u}{\partial t}$, we obtain

$$
\begin{aligned}
& v\left(\alpha^{+}, t\right)=v\left(\alpha^{-}, t\right)+\frac{1}{K} \frac{\partial}{\partial t} \sigma\left(\alpha^{-}, t\right) \\
& \sigma\left(\alpha^{+}, t\right)=\sigma\left(\alpha^{-}, t\right)+M \frac{\partial}{\partial t} v\left(\alpha^{-}, t\right) .
\end{aligned}
$$

The time derivatives in (3.3) are replaced by spatial derivatives via the conservation laws (2.2), hence

$$
\begin{aligned}
v\left(\alpha^{+}, t\right) & =v\left(\alpha^{-}, t\right)+\frac{\rho_{0} c_{0}^{2}}{K} \frac{\partial}{\partial x} v\left(\alpha^{-}, t\right) \\
\sigma\left(\alpha^{+}, t\right) & =\sigma\left(\alpha^{-}, t\right)+\frac{M}{\rho_{0}} \frac{\partial}{\partial x} \sigma\left(\alpha^{-}, t\right) .
\end{aligned}
$$

Setting

$$
\boldsymbol{S}_{0}=\operatorname{diag}\left(\frac{\rho_{0} c_{0}^{2}}{K}, \frac{M}{\rho_{0}}\right)
$$

we get

$$
[\boldsymbol{U}(\alpha, t)]=\boldsymbol{S}_{0} \frac{\partial}{\partial x} \boldsymbol{U}\left(\alpha^{-}, t\right)
$$

The next two results express $\boldsymbol{U}(x, t)$ and its spatial derivatives on one side of $\alpha$ in terms of $\boldsymbol{U}(x, t)$ and its spatial derivatives on the other side of $\alpha$.

RESULT 2. Limit values of $\frac{\partial^{k}}{\partial x^{k}} \boldsymbol{U}(x, t)$ on the right of $\alpha$ are given for all $k \geq 0$ and for all time $t$ by

$$
\frac{\partial^{k}}{\partial x^{k}} \boldsymbol{U}\left(\alpha^{+}, t\right)=\boldsymbol{D}_{2 k+1} \frac{\partial^{k}}{\partial x^{k}} \boldsymbol{U}\left(\alpha^{-}, t\right)+\boldsymbol{D}_{2 k+2} \frac{\partial^{k+1}}{\partial x^{k+1}} \boldsymbol{U}\left(\alpha^{-}, t\right),
$$


with matrices $\boldsymbol{D}_{2 k+1}$ and $\boldsymbol{D}_{2 k+2}$ depending on whether $k$ is odd or even

$$
\begin{aligned}
& \boldsymbol{D}_{4 i+1}=\operatorname{diag}\left(\left(\frac{c_{0}}{c_{1}}\right)^{2 i},\left(\frac{c_{0}}{c_{1}}\right)^{2 i}\right), \\
& \boldsymbol{D}_{4 i+2}=\operatorname{diag}\left(\frac{\rho_{0}}{K} \frac{\left(c_{0}\right)^{2 i+2}}{\left(c_{1}\right)^{2 i}}, \frac{M}{\rho_{0}}\left(\frac{c_{0}}{c_{1}}\right)^{2 i}\right), \\
& \boldsymbol{D}_{4 i+3}=\operatorname{diag}\left(\frac{\rho_{0}}{\rho_{1}}\left(\frac{c_{0}}{c_{1}}\right)^{2 i+2}, \frac{\rho_{1}}{\rho_{0}}\left(\frac{c_{0}}{c_{1}}\right)^{2 i}\right), \\
& \boldsymbol{D}_{4 i+4}=\operatorname{diag}\left(\frac{M}{\rho_{1}}\left(\frac{c_{0}}{c_{1}}\right)^{2 i+2}, \frac{\rho_{1}}{K} \frac{\left(c_{0}\right)^{2 i+2}}{\left(c_{1}\right)^{2 i}}\right) .
\end{aligned}
$$

Proof. Since (3.5) is true for all time $t$, we differentiate (3.5) $2 i$-times with respect to $t$ and we substitute the first equation of (2.5) to obtain

$$
\left[c^{2 i} \frac{\partial^{2 i}}{\partial x^{2 i}} \boldsymbol{U}\right]=c_{0}^{2 i} \boldsymbol{S} \frac{\partial^{2 i+1}}{\partial x^{2 i+1}} \boldsymbol{U}\left(\alpha^{-}, t\right) .
$$

We also differentiate (3.5) $2 i+1$-times with respect to $t$ and we substitute the second equation of $(2.5)$ to get

$$
\left[-c^{2 i} A \frac{\partial^{2 i+1}}{\partial x^{2 i+1}} \boldsymbol{U}\right]=-c_{0}^{2 i} \boldsymbol{S} \boldsymbol{A}_{0} \frac{\partial^{2 i+2}}{\partial x^{2 i+2}} \boldsymbol{U}\left(\alpha^{-}, t\right) .
$$

Simple algebric manipulations on (3.7) and (3.8) give (3.6).

RESUlt 3. Limit values of $\frac{\partial^{k}}{\partial x^{k}} \boldsymbol{U}(x, t)$ on the left of $\alpha$ are given for all $k \geq 0$, $l \geq 1$, and for all time $t$ by

$$
\frac{\partial^{k}}{\partial x^{k}} \boldsymbol{U}\left(\alpha^{-}, t\right)=\sum_{m=0}^{l-1} \boldsymbol{E}(m, k, l) \frac{\partial^{k+m}}{\partial x^{k+m}} \boldsymbol{U}\left(\alpha^{+}, t\right)+\boldsymbol{E}(l, k, l) \frac{\partial^{k+l}}{\partial x^{k+l}} \boldsymbol{U}\left(\alpha^{-}, t\right),
$$

with

$$
\begin{aligned}
& m<l \Rightarrow \boldsymbol{E}(m, k, l)=(-1)^{m}\left(\boldsymbol{D}_{2 k+1}\right)^{-1} \prod_{j=1}^{m} \boldsymbol{D}_{2(k+j)}\left(\boldsymbol{D}_{2(k+j)+1}\right)^{-1} \\
& m=l \Rightarrow \boldsymbol{E}(l, k, l)=(-1)^{l}\left(\boldsymbol{D}_{2 k+1}\right)^{-1}\left(\prod_{j=1}^{l-1} \boldsymbol{D}_{2(k+j)}\left(\boldsymbol{D}_{2(k+j)+1}\right)^{-1}\right) \boldsymbol{D}_{2(k+l)},
\end{aligned}
$$

and with the convention

$$
\prod_{j=1}^{0} \boldsymbol{D}_{2(k+j)}\left(\boldsymbol{D}_{2(k+j)+1}\right)^{-1}=\boldsymbol{I}_{2}
$$

where $\boldsymbol{I}_{2}$ is the identity matrix.

Proof. The proof is performed by induction on $l$. From (3.6), we write for all integer $j \geq 0$

$$
\frac{\partial^{j}}{\partial x^{j}} \boldsymbol{U}\left(\alpha^{-}, t\right)=\left(\boldsymbol{D}_{2 j+1}\right)^{-1} \frac{\partial^{j}}{\partial x^{j}} \boldsymbol{U}\left(\alpha^{+}, t\right)-\left(\boldsymbol{D}_{2 j+1}\right)^{-1} \boldsymbol{D}_{2 j+2} \frac{\partial^{j+1}}{\partial x^{j+1}} \boldsymbol{U}\left(\alpha^{-}, t\right) .
$$


Taking $j=k$ in (3.11) induces the relation (3.9) for $l=1$. Then, suppose that (3.9) holds for $l \geq 1$. We write (3.11) for $j=k+l$ and we insert the result in (3.9). The definitions of matrices $\boldsymbol{E}$ lead to the relation (3.9) for $l+1$.

REMARK 1. Note that spatial derivatives of $\boldsymbol{U}$ on the left side cannot be expressed only in terms of spatial derivatives of $\boldsymbol{U}$ on the right side.

Some values of $M$ and $K$ lead to classical cases of contact.

1. If $M=0$ and $K \rightarrow+\infty$, we get $\boldsymbol{D}_{2 k+2}=\mathbf{0}$. Then conditions (3.6) and (3.9) recover conditions for perfect interfaces examined in [23].

2. If $M \neq 0$ or $K<+\infty$, we get $\boldsymbol{D}_{2 k+2} \neq 0$ even if $\rho_{0}=\rho_{1}$ and $c_{0}=c_{1}$, hence there is still a jump of $\frac{\partial^{k}}{\partial x^{k}} \boldsymbol{U}(x, t)$ across $\alpha$. This is obviously not the case for perfect interfaces. Physically, this situation corresponds to the idealised case of a glue layer between identical materials. It is investigated numerically in 5 .

3. The case of a homogeneous medium, i.e. where the interface actually disappears, is obtained only for $\rho_{0}=\rho_{1}, c_{0}=c_{1}, M=0$, and $K \rightarrow+\infty$.

\section{Insertion of the spring-mass conditions in numerical schemes.}

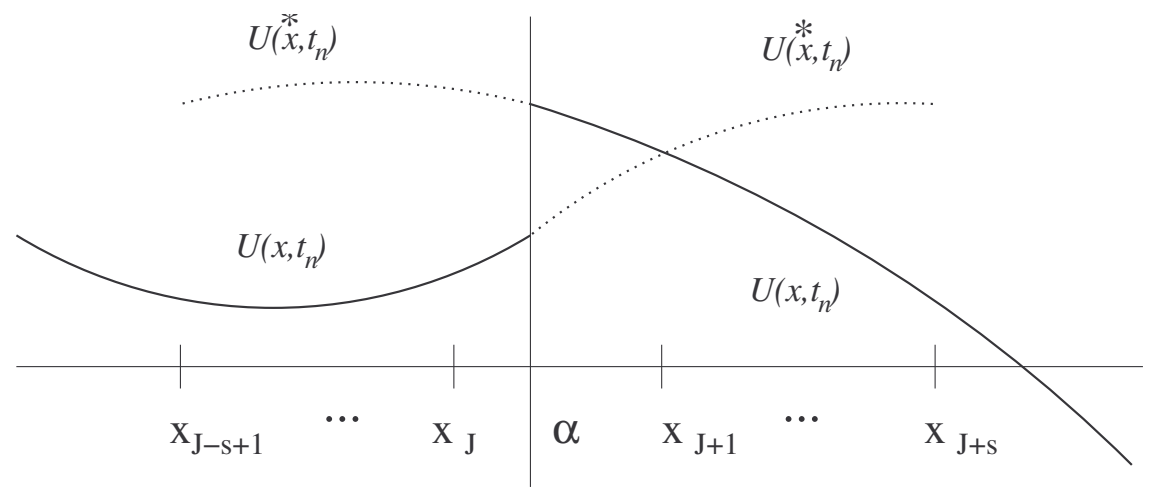

FIG. 4.1. Exact solution $\boldsymbol{U}\left(x, t_{n}\right)$ (solid line) and the modified solutions $\boldsymbol{U}^{*}\left(x, t_{n}\right)$ (dotted line).

4.1. The interface method. We recall the key points of the Explicit Simplified Interface Method (ESIM) [23, 19]. Firstly, we define smooth extensions $\boldsymbol{U}^{*}\left(x, t_{n}\right)$ of the exact solution $\boldsymbol{U}\left(x, t_{n}\right)$ on both sides of $\alpha$ at each time step $t_{n}$ (Fig. 4.1). The extension $\boldsymbol{U}^{*}\left(x, t_{n}\right)$ satisfies exactly the same conditions than $\boldsymbol{U}\left(x, t_{n}\right)$ at $\alpha$. Secondly, numerical approximations of these extensions are computed at the irregular points $x_{i}$ $(i=J-s+1, \ldots, J+s)$; they are called modified values and they are written $\boldsymbol{U}_{i}^{*}$ (see 4.1.1 and 4.1.2). Thirdly, at an irregular point on one side of $\alpha$, the time-stepping of the chosen scheme classically uses numerical values on this side, and modified values on the other side (see 4.1.3).

We write the smooth extensions as polynomials in $x$ of degree $2 k-1$

$$
\begin{aligned}
& \text { for } x>\alpha, \quad \boldsymbol{U}^{*}\left(x, t_{n}\right)=\sum_{m=0}^{2 k-1} \frac{(x-\alpha)^{m}}{m !} \frac{\partial^{m}}{\partial x^{m}} \boldsymbol{U}\left(\alpha^{-}, t_{n}\right), \\
& \text { for } x \leq \alpha, \quad \boldsymbol{U}^{*}\left(x, t_{n}\right)=\sum_{m=0}^{2 k-1} \frac{(x-\alpha)^{m}}{m !} \frac{\partial^{m}}{\partial x^{m}} \boldsymbol{U}\left(\alpha^{+}, t_{n}\right) .
\end{aligned}
$$


The required value of the integer $k$ depends on the order of the chosen scheme. We show in 4.4 . that $k=2$ is required e.g. for the Lax-Wendroff scheme. The initial data $\boldsymbol{U}_{0}(x)$ is supposed to be sufficiently smooth so that $\boldsymbol{U}\left(x, t_{n}\right)$ and its spatial derivatives up to the $(2 k-1)$-th order are well-defined outside $\alpha$, and at $\alpha^{-}$and $\alpha^{+}$.

The goal of the next two sections is to calculate estimations of $\boldsymbol{U}^{*}\left(x, t_{n}\right)$ at the irregular points on the right $(i=J+1, \ldots . J+s)$ in terms of $\frac{\partial^{m}}{\partial x^{m}} \boldsymbol{U}\left(\alpha^{-}, t_{n}\right)$, and on the left $(i=J-s+1, \ldots, J)$ in terms of $\frac{\partial^{k}}{\partial x^{m}} \boldsymbol{U}\left(\alpha^{+}, t_{n}\right)$. For that purpose, Taylor expansions of the exact solution $\boldsymbol{U}\left(x, t_{n}\right)$ at the $2 k$ grid points $x_{i}$ surrounding the interface are written around $\alpha^{ \pm}$.

$$
\begin{aligned}
& i=J-k+1, \ldots, J, \quad \boldsymbol{U}\left(x_{i}, t_{n}\right)=\sum_{m=0}^{2 k-1} \frac{\left(x_{i}-\alpha\right)^{m}}{m !} \frac{\partial^{m}}{\partial x^{m}} \boldsymbol{U}\left(\alpha^{-}, t_{n}\right)+\boldsymbol{O}\left(\Delta x^{2 k}\right) \\
& i=J+1, \ldots, J+k, \quad \boldsymbol{U}\left(x_{i}, t_{n}\right)=\sum_{m=0}^{2 k-1} \frac{\left(x_{i}-\alpha\right)^{m}}{m !} \frac{\partial^{m}}{\partial x^{m}} \boldsymbol{U}\left(\alpha^{+}, t_{n}\right)+\boldsymbol{O}\left(\Delta x^{2 m}\right)
\end{aligned}
$$

Throughout this paper and depending on the context, the notation $O\left(\Delta x^{\lambda}\right)$, generally used for a scalar, refers also to a vector with two components, or to a 2-by-2 diagonal matrix, of which the entries are $O\left(\Delta x^{\lambda}\right)$ scalars.

4.1.1. Modified values on the right. We substitute the conditions (3.6) in (4.4). Then we get at grid points on the right $(i=J+1, \ldots, J+k)$

$$
\begin{aligned}
\boldsymbol{U}\left(x_{i}, t_{n}\right) & =\sum_{m=0}^{2 k-1} \frac{\left(x_{i}-\alpha\right)^{m}}{m !} \boldsymbol{D}_{2 m+1} \frac{\partial^{m}}{\partial x^{m}} \boldsymbol{U}\left(\alpha^{-}, t_{n}\right) \\
& +\sum_{m=0}^{2 k-1} \frac{\left(x_{i}-\alpha\right)^{m}}{m !} \boldsymbol{D}_{2 m+2} \frac{\partial^{m+1}}{\partial x^{m+1}} \boldsymbol{U}\left(\alpha^{-}, t_{n}\right)+O\left(\Delta x^{2 k}\right),
\end{aligned}
$$

or, after grouping together the sums in (4.5),

$$
\begin{aligned}
\boldsymbol{U}\left(x_{i}, t_{n}\right) & =\boldsymbol{D}_{1} \boldsymbol{U}\left(\alpha^{-}, t_{n}\right) \\
& +\sum_{m=1}^{2 k-1}\left(\frac{\left(x_{i}-\alpha\right)^{m}}{m !} \boldsymbol{D}_{2 m+1}+\frac{\left(x_{i}-\alpha\right)^{m-1}}{(m-1) !} \boldsymbol{D}_{2 m}\right) \frac{\partial^{m}}{\partial x^{m}} \boldsymbol{U}\left(\alpha^{-}, t_{n}\right) \\
& +\frac{\left(x_{i}-\alpha\right)^{2 k-1}}{(2 k-1) !} \boldsymbol{D}_{4 k} \frac{\partial^{2 k}}{\partial x^{2 k}} \boldsymbol{U}\left(\alpha^{-}, t_{n}\right)+\boldsymbol{O}\left(\Delta x^{2 k}\right) .
\end{aligned}
$$

The relations (4.3) and (4.6) are summed up as follows

$$
\left(\begin{array}{c}
\boldsymbol{U}\left(x_{J-k+1}, t_{n}\right) \\
\vdots \\
\boldsymbol{U}\left(x_{J+k}, t_{n}\right)
\end{array}\right)=\boldsymbol{M}\left(\begin{array}{c}
\boldsymbol{U}\left(\alpha^{-}, t_{n}\right) \\
\vdots \\
\frac{\partial^{2 k-1}}{\partial x^{2 k-1}} \boldsymbol{U}\left(\alpha^{-}, t_{n}\right)
\end{array}\right)+\boldsymbol{\Delta}_{\mathbf{1}}+\left(\begin{array}{c}
\boldsymbol{O}\left(\Delta x^{2 k}\right) \\
\vdots \\
\boldsymbol{O}\left(\Delta x^{2 k}\right)
\end{array}\right)
$$


where $\boldsymbol{M}$ is a $2 k$-by- $2 k$ block matrix with 2 -by- 2 blocks, and with entries $(j=$ $1, \ldots, 2 k)$

$$
\boldsymbol{M}[i, j]=\left\{\begin{array}{l}
\frac{\left(x_{J-k+i}-\alpha\right)^{j-1}}{(j-1) !} \boldsymbol{I}_{2} \quad \text { if } 1 \leq i \leq k, \\
\boldsymbol{D}_{1} \quad \text { if } k+1 \leq i \leq 2 k \text { and if } j=1, \\
\frac{\left(x_{J-k+i}-\alpha\right)^{j-1}}{(j-1) !} \boldsymbol{D}_{2 j-1}+\frac{\left(x_{J-k+i}-\alpha\right)^{j-2}}{(j-2) !} \boldsymbol{D}_{2 j-2} \\
\text { if } k+1 \leq i \leq 2 k \text { and if } j \neq 1 .
\end{array}\right.
$$

$\boldsymbol{\Delta}_{\mathbf{1}}$ is a $2 k$ block vector with entries

$$
\boldsymbol{\Delta}_{1}[i]=\left\{\begin{array}{l}
0 \quad \text { if } 1 \leq i \leq k, \\
\frac{\left(x_{J-k+i}-\alpha\right)^{2 k-1}}{(2 k-1) !} \boldsymbol{D}_{4 k} \frac{\partial^{2 k}}{\partial x^{2 k}} \boldsymbol{U}\left(\alpha^{-}, t_{n}\right) \quad \text { if } k+1 \leq i \leq 2 k .
\end{array}\right.
$$

To obtain estimations of $\frac{\partial^{m}}{\partial x^{m}} \boldsymbol{U}\left(\alpha^{-}, t_{n}\right)(m=0, \ldots, 2 k-1)$, we replace the exact values $\boldsymbol{U}\left(x_{i}, t_{n}\right)$ in (4.7) by the numerical values $\boldsymbol{U}_{i}^{n}$, we eliminate both $\boldsymbol{\Delta}_{1}$ and the vector of truncation errors, and we invert the resulting system. Then, estimations of $\boldsymbol{U}^{*}\left(x_{i}, t_{n}\right)$ at irregular points on the right of $\alpha$ are deduced from (4.1) and (4.7)

$$
i=J+1, \ldots, J+s, \quad \boldsymbol{U}_{i}^{*}=\left(\boldsymbol{I}_{2}, \ldots, \frac{\left(x_{i}-\alpha\right)^{2 k-1}}{(2 k-1) !} \boldsymbol{I}_{2}\right) \boldsymbol{M}^{-1}\left(\begin{array}{c}
\boldsymbol{U}_{J-k+1}^{n} \\
\vdots \\
\boldsymbol{U}_{J+k}^{n}
\end{array}\right) .
$$

4.1.2. Modified values on the left. We follow a similar procedure to compute the modified values on the left side. We substitute the conditions (3.9) calculated up to $l=2 k-m(m=0, \ldots, 2 k-1)$ in $(4.3)$ and we get, at grid points on the left $(i=J-k+1, \ldots, J)$,

$$
\begin{aligned}
\boldsymbol{U}\left(x_{i}, t_{n}\right) & =\sum_{m=0}^{2 k-1} \sum_{\lambda=0}^{2 k-m-1} \frac{\left(x_{i}-\alpha\right)^{m}}{m !} \boldsymbol{E}(\lambda, k, 2 k-m) \frac{\partial^{\lambda+m}}{\partial x^{\lambda+m}} \boldsymbol{U}\left(\alpha^{+}, t_{n}\right) \\
& +\sum_{m=0}^{2 k-1} \frac{\left(x_{i}-\alpha\right)^{m}}{m !} \boldsymbol{E}(2 k-m, m, 2 k-m) \frac{\partial^{2 k}}{\partial x^{2 k}} \boldsymbol{U}\left(\alpha^{-}, t_{n}\right)+\boldsymbol{O}\left(\Delta x^{2 k}\right),
\end{aligned}
$$

or, after a rearrangement of the double sum,

$$
\begin{aligned}
\boldsymbol{U}\left(x_{i}, t_{n}\right) & =\sum_{m=0}^{2 k-1}\left(\sum_{\lambda=0}^{m} \frac{\left(x_{i}-\alpha\right)^{m-\lambda}}{(m-\lambda) !} \boldsymbol{E}(\lambda, m-\lambda, 2 k-m+\lambda)\right) \frac{\partial^{m}}{\partial x^{m}} \boldsymbol{U}\left(\alpha^{+}, t_{n}\right) \\
& +\left(\sum_{m=0}^{2 k-1} \frac{\left(x_{i}-\alpha\right)^{m}}{m !} \boldsymbol{E}(2 k-m, m, 2 k-m)\right) \frac{\partial^{2 k}}{\partial x^{2 k}} \boldsymbol{U}\left(\alpha^{-}, t_{n}\right)+\boldsymbol{O}\left(\Delta x^{2 k}\right) .
\end{aligned}
$$


Then, the relations (4.4) and (4.12) are summed up as follows

$$
\left(\begin{array}{c}
\boldsymbol{U}\left(x_{J-k+1}, t_{n}\right) \\
\vdots \\
\boldsymbol{U}\left(x_{J+k}, t_{n}\right)
\end{array}\right)=\boldsymbol{N}\left(\begin{array}{c}
\boldsymbol{U}\left(\alpha^{-}, t_{n}\right) \\
\vdots \\
\frac{\partial^{2 k-1}}{\partial x^{2 k-1}} \boldsymbol{U}\left(\alpha^{-}, t_{n}\right)
\end{array}\right)+\boldsymbol{\Delta}_{\mathbf{2}}+\left(\begin{array}{c}
\boldsymbol{O}\left(\Delta x^{2 k}\right) \\
\vdots \\
\boldsymbol{O}\left(\Delta x^{2 k}\right)
\end{array}\right)
$$

where $\boldsymbol{N}$ is a $2 k$-by- $2 k$ block matrix with 2 -by-2 blocks, and with entries $(j=$ $1, \ldots, 2 k)$

$\boldsymbol{N}[i, j]=\left\{\begin{array}{l}\sum_{m=0}^{j-1} \frac{\left(x_{J-k+i}-\alpha\right)^{j-1-m}}{(j-1-m) !} \boldsymbol{E}(m, j-1-m, 2 k-j+1+m) \text { if } 1 \leq i \leq k, \\ \frac{\left(x_{J-k+i}-\alpha\right)^{j-1}}{(j-1) !} \boldsymbol{I}_{2} \text { if } k+1 \leq i \leq 2 k .\end{array}\right.$

$\boldsymbol{\Delta}_{2}$ is a $2 k$ block vector with entries

$$
\boldsymbol{\Delta}_{2}[i]=\left\{\begin{array}{l}
\left(\sum_{m=0}^{2 k-1} \frac{\left(x_{J-k+i}-\alpha\right)^{m}}{m !} \boldsymbol{E}(2 k-m, m, 2 k-m)\right) \frac{\partial^{2 k}}{\partial x^{2 k}} \boldsymbol{U}\left(\alpha^{-}, t_{n}\right) \text { if } 1 \leq i \leq k, \\
0 \text { if } k+1 \leq i \leq 2 k .
\end{array}\right.
$$

Estimations of $\boldsymbol{U}^{*}\left(x_{i}, t_{n}\right)$ at the irregular points on the left of $\alpha$ are deduced from (4.2) and (4.13) after eliminating both $\boldsymbol{\Delta}_{2}$ and the vector of truncation errors

$$
i=J-s+1, \ldots, J, \quad \boldsymbol{U}_{i}^{*}=\left(\boldsymbol{I}_{2}, \ldots, \frac{\left(x_{i}-\alpha\right)^{2 k-1}}{(2 k-1) !} \boldsymbol{I}_{2}\right) \boldsymbol{N}^{-1}\left(\begin{array}{c}
\boldsymbol{U}_{J-k+1}^{n} \\
\vdots \\
\boldsymbol{U}_{J+k}^{n}
\end{array}\right)
$$

4.2. Time-stepping at the irregular points. Instead of (2.6), the timestepping of a two-stage scheme at the irregular points $x_{i}(i=J-s+1, \ldots, J+s)$ uses modified values on the side other than that for $x_{i}$

$$
\begin{array}{ll}
J-s+1 \leq i \leq J, & \boldsymbol{U}_{i}^{n+1}=\boldsymbol{U}_{i}^{n}+\boldsymbol{H}\left(\boldsymbol{U}_{i-s}^{n}, \ldots, \boldsymbol{U}_{J}^{n}, \boldsymbol{U}_{J+1}^{*}, \ldots, \boldsymbol{U}_{i+s}^{*}\right) \\
J+1 \leq i \leq J+s, & \boldsymbol{U}_{i}^{n+1}=\boldsymbol{U}_{i}^{n}+\boldsymbol{H}\left(\boldsymbol{U}_{i-s}^{*}, \ldots, \boldsymbol{U}_{J}^{*}, \boldsymbol{U}_{J+1}^{n}, \ldots, \boldsymbol{U}_{i+s}^{n}\right) .
\end{array}
$$

Then, the finite-difference scheme is said to be coupled with the ESIM $k$.The coupling of the ESIM with four-stage schemes like WENO 5 is obvious: we apply (4.17) at each step of Runge-Kutta integrations between $t_{n}$ and $t_{n+1}$. A similar procedure is easy to adapt also to classical, multistep, and explicit finite-difference schemes used to integrate the second-order scalar elastic wave equation.

The following result guarantees that the modified values are well-defined for the schemes used throughout this study.

RESUlt 4. Matrices $\boldsymbol{M}$ and $\boldsymbol{N}$ are always invertible if $k=1,2,3$, whatever $\Delta x$ and the position of $\alpha$ in the interval $\left[x_{J}, x_{J+1}[\right.$.

Proof. The proof is proposed only for $k=2$, and it has been verified for $k=1$ and $k=3$. Let us consider the case of $\boldsymbol{M}$. We write $\theta=\left(\alpha-x_{J}\right) / \Delta x$, so $\theta \in[0,1[$. 
The blocks of $\boldsymbol{M}$ (4.8) are diagonal: by permuting lines and rows, we obtain

$$
\operatorname{det} \boldsymbol{M}=\operatorname{det} \boldsymbol{A} \operatorname{det} \boldsymbol{B},
$$

where $\boldsymbol{A}[i, j]=\boldsymbol{M}[i, j][1,1]$ and $\boldsymbol{B}[i, j]=\boldsymbol{M}[i, j][2,2](i=1, \ldots, 4, j=1, \ldots, 4)$. Computations with MAPLE show that

$\operatorname{det} \boldsymbol{A}=\frac{1}{12 \rho_{1}^{2} c_{1}^{2} K^{2}}\left(\left(\sum_{i=1}^{5} \eta_{i} p_{i}(\theta)\right) \Delta x^{6}+\left(\sum_{i=6}^{9} \eta_{i} p_{i}(\theta)\right) \Delta x^{5}+\left(\sum_{i=10}^{12} \eta_{i} p_{i}(\theta)\right) \Delta x^{4}\right)$

$\operatorname{det} \boldsymbol{B}=\frac{1}{12 \rho_{0}^{2} c_{1}^{4} K}\left(\left(\sum_{i=1}^{5} \xi_{i} p_{i}(\theta)\right) \Delta x^{6}+\left(\sum_{i=6}^{9} \xi_{i} p_{i}(\theta)\right) \Delta x^{5}+\left(\sum_{i=10}^{12} \xi_{i} p_{i}(\theta)\right) \Delta x^{4}\right)$

with the polynomials $p_{i}(\theta)$

$$
\begin{array}{lll}
p_{1}(\theta)=-4 \theta^{4}+16 \theta^{3}-17 \theta^{2}-\theta+6, & p_{7}(\theta)=-12 \theta^{3}+6 \theta^{2}+16 \theta+3, \\
p_{2}(\theta)=\theta^{4}-6 \theta^{3}+13 \theta^{2}-12 \theta+4, & p_{8}(\theta) & =4 \theta^{3}+6 \theta^{2}+2 \theta, \\
p_{3}(\theta)=6 \theta^{4}-12 \theta^{3}-4 \theta^{2}+10 \theta+2, & p_{9}(\theta) & =-4 \theta^{3}+18 \theta^{2}-26 \theta+12, \\
p_{4}(\theta)=\theta^{4}+2 \theta^{3}+\theta^{2}, & p_{10}(\theta)=6 \theta^{2}+6 \theta+2, \\
p_{5}(\theta)=-4 \theta^{4}+7 \theta^{2}+3 \theta, & p_{11}(\theta)=6 \theta^{2}-18 \theta+12, \\
p_{6}(\theta)=12 \theta^{3}-30 \theta^{2}+8 \theta+13, & p_{12}(\theta) & =-12 \theta^{2}+12 \theta+9,
\end{array}
$$

and the coefficients $\eta_{i}$ et $\xi_{i}$

$$
\begin{aligned}
& \eta_{1}=\rho_{0}^{2} c_{0}^{6} K^{2}, \quad \eta_{2}=\rho_{0} c_{0}^{6} \rho_{1} K^{2}, \quad \eta_{3}=\rho_{0} c_{0}^{4} \rho_{1} c_{1}^{2} K^{2}, \\
& \eta_{4}=\rho_{0} c_{0}^{2} \rho_{1} c_{1}^{4} K^{2} \quad \eta_{5}=c_{0}^{2} \rho_{1}^{2} c_{1}^{4} K^{2}, \quad \eta_{6} \quad=\rho_{0}^{2} c_{0}^{6} \rho_{1} c_{1}^{2} K, \\
& \eta_{7}=\rho_{0} c_{0}^{4} \rho_{1}^{2} c_{1}^{4} K \quad \eta_{8} \quad=\rho_{1} c_{1}^{6} K^{2} M, \quad \eta_{9} \quad=\rho_{0} c_{0}^{4} c_{1}^{2} K^{2} M, \\
& \eta_{10}=\rho_{0} c_{0}^{2} \rho_{1} c_{1}^{6} K M, \quad \eta_{11}=\rho_{0} c_{0}^{4} \rho_{1} c_{1}^{4} K M, \quad \eta_{12}=\rho_{0}^{2} c_{0}^{6} \rho_{1}^{2} c_{1}^{4}, \\
& \xi_{1}=\rho_{0} c_{0}^{4} \rho_{1} K, \quad \xi_{2}=\rho_{0} c_{0}^{2} \rho_{1} c_{1}^{2} K, \quad \xi_{3} \quad=\rho_{1}^{2} c_{0}^{2} c_{1}^{2} K, \\
& \xi_{4}=\rho_{0} \rho_{1} c_{1}^{4} K, \quad \xi_{5}=\rho_{0}^{2} c_{0}^{2} c_{1}^{2} K, \quad \xi_{9}=\rho_{0} c_{0}^{4} \rho_{1}^{2} c_{1}^{2}, \\
& \xi_{7}=\rho_{0} c_{0}^{2} c_{1}^{2} K M, \quad \xi_{8} \quad=\rho_{0}^{2} c_{0}^{2} \rho_{1} c_{1}^{4}, \quad \xi_{6} \quad=c_{0}^{2} \rho_{1} c_{1}^{2} K M, \\
& \xi_{10}=\rho_{0} c_{0}^{2} \rho_{1} c_{1}^{4} M, \quad \xi_{11}=\rho_{0} c_{0}^{4} \rho_{1} c_{1}^{2} M, \quad \xi_{12}=c_{0}^{2} c_{1}^{2} K M^{2} .
\end{aligned}
$$

We observe that

$$
\sum_{i=1}^{12} p_{i}(\theta)=63
$$


The polynomials $p_{i}$ and $q_{i}$ satisfy $p_{i}(\theta) \geq 0$ and $q_{i}(\theta) \geq 0$ on $[0,1]$, and they are not all equal to zero simultaneously. Since $\eta_{i}>0, \xi_{i}>0(i=1, \ldots, 12)$, we find that $\operatorname{det} \boldsymbol{A}>0$ and $\operatorname{det} \boldsymbol{B}>0$. Furthermore, even for $K \rightarrow+\infty$, the $1 / K^{2}$ and $1 / K$ factors in (4.19) are simplified by coefficients $\eta_{i}$ in $K^{2}$ and $\xi_{i}$ in $K$, hence $\operatorname{det} \boldsymbol{A}$ and $\operatorname{det} \boldsymbol{B}$ are still different from zero. In all cases, it implies $\operatorname{det} \boldsymbol{M}>0$. The same work has been done for $\boldsymbol{N}$, giving also $\operatorname{det} \boldsymbol{N}>0$.

Note that the matrices $\boldsymbol{M}^{-1}$ and $\boldsymbol{N}^{-1}$ need to be computed only once, during a preprocessing step. At each time step, only the matrix-vector multiplications (4.10) and (4.16) need to be done. The computational cost therefore remains much lower than that for time-stepping.

We do not propose an analysis of the stability of (4.17). Numerous experiments have shown no influence of $K$ and $M$ on the stability of the coupling of numerical schemes with the ESIM $k$, whatever the position of $\alpha$ in the interval $\left[x_{J}, x_{J+1}[\right.$.

Result 5. Consider the limit case of a homogeneous medium, i.e. $\rho_{0}=\rho_{1}, c_{0}=$ $c_{1}, K=+\infty$ and $M=0$. Then the coupling of a numerical scheme with the ESIM $k$ is consistent with the numerical scheme in homogeneous medium, i.e. the time-stepping (4.17) recovers the time-stepping (2.6), if

$$
k \geq s .
$$

Proof. In the limit case of a homogeneous medium, matrices $D$ and $E$ used in (3.6) and (3.9) are simplified, and we get for all $i \geq 0, k \geq 0$, and $l \geq 1$

$$
\left\{\begin{array}{l}
\boldsymbol{D}_{4 i+1}=\boldsymbol{D}_{4 i+3}=\boldsymbol{I}_{2} \\
\boldsymbol{D}_{4 i+2}=\boldsymbol{D}_{4 i+4}=\mathbf{0} \\
\boldsymbol{E}(0, k, l)=\boldsymbol{I}_{2} \text { and } \boldsymbol{E}(m, k, l)=\mathbf{0} \text { if } m \neq 0 .
\end{array}\right.
$$

As a consequence, $\boldsymbol{M}$ and $\boldsymbol{N}$ are now equal. Therefore, (4.10) and (4.16) can be written both for right-sided and left-sided irregular points

$$
J-s+1 \leq i \leq J+s, \quad \boldsymbol{U}_{i}^{*}=\left(1, \ldots, \frac{\left(x_{i}-\alpha\right)^{2 k-1}}{(2 k-1) !}\right) \boldsymbol{M}^{-1}\left(\begin{array}{c}
\boldsymbol{U}_{J-k+1}^{n} \\
\vdots \\
\boldsymbol{U}_{J+k}^{n}
\end{array}\right) .
$$

Moreover, $\boldsymbol{M}$ is simplified and we get

$$
\begin{aligned}
\left(\begin{array}{c}
\boldsymbol{U}_{J-k+1}^{n} \\
\vdots \\
\boldsymbol{U}_{J+k}^{n}
\end{array}\right) & =\boldsymbol{M} \boldsymbol{M}^{-1}\left(\begin{array}{c}
\boldsymbol{U}_{J-k+1}^{n} \\
\vdots \\
\boldsymbol{U}_{J+k}^{n}
\end{array}\right) \\
& =\left(\begin{array}{ccc}
\boldsymbol{I}_{2} & \ldots & \frac{\left(x_{J-k+1}-\alpha\right)^{2 k-1}}{(2 k-1) !} \boldsymbol{I}_{2} \\
\vdots & & \vdots \\
\boldsymbol{I}_{2} & \ldots & \frac{\left(x_{J+k}-\alpha\right)^{2 k-1}}{(2 k-1) !} \boldsymbol{I}_{2}
\end{array}\right) \boldsymbol{M}^{-1}\left(\begin{array}{c}
\boldsymbol{U}_{J-k+1}^{n} \\
\vdots \\
\boldsymbol{U}_{J+k}^{n}
\end{array}\right)
\end{aligned}
$$


which yields

$$
J-k+1 \leq i \leq J+k, \quad \boldsymbol{U}_{i}^{n}=\left(\boldsymbol{I}_{2}, \ldots, \frac{\left(x_{i}-\alpha\right)^{2 k-1}}{(2 k-1) !} \boldsymbol{I}_{2}\right) \boldsymbol{M}^{-1}\left(\begin{array}{c}
\boldsymbol{U}_{J-k+1}^{n} \\
\vdots \\
\boldsymbol{U}_{J+k}^{n}
\end{array}\right)
$$

Comparing (4.21) and (4.23) leads to

$$
k \geq s \quad \Rightarrow \quad J-s+1 \leq i \leq J+s, \quad \boldsymbol{U}_{i}^{*}=\boldsymbol{U}_{i}^{n} .
$$

In this case, we deduce from (4.17) that the time-stepping in homogeneous medium (2.6) is completely recovered.

4.3. Local truncation analysis. In [23], we analysed the local truncation error of the coupling (4.17) for the perfect conditions (3.1) and for $k \leq 3$. To apply the same analysis for the spring-mass conditions (3.2) and for $k \leq 3$, we need to take into account the new structure of block vectors and block matrices (4.7) and (4.13). For the perfect conditions (3.1) indeed, we have $\boldsymbol{\Delta}_{1}=\boldsymbol{\Delta}_{2}=\mathbf{0}$, and since for all $i$, $\left|x_{i}-\alpha\right|=O(\Delta x)$, we have

$$
M[i, j]=O\left(\Delta x^{j-1}\right), \quad \boldsymbol{N}[i, j]=O\left(\Delta x^{j-1}\right)
$$

for $i=1, \ldots, 2 k$ and $j=1, \ldots, 2 k$. For the spring-mass conditions (3.2), $\boldsymbol{\Delta}_{1}$ and $\boldsymbol{\Delta}_{2}$ are non-zero vectors, and we have

$$
\begin{aligned}
& \boldsymbol{M}[i, j]=\left\{\begin{array}{l}
O\left(\Delta x^{j-1}\right) \text { if } 1 \leq i \leq k \text { and } 1 \leq j \leq 2 k, \\
O(1) \text { if } k+1 \leq i \leq 2 k \text { and } j=1, \\
O\left(\Delta x^{j-2}\right) \text { if } k+1 \leq i \leq 2 k \text { and } 2 \leq j \leq 2 k,
\end{array}\right. \\
& \boldsymbol{N}[i, j]=\left\{\begin{array}{l}
O(1) \text { if } 1 \leq i \leq k \text { and } 1 \leq j \leq 2 k, \\
O\left(\Delta x^{j-1}\right) \text { if } k+1 \leq i \leq 2 k \text { and } 1 \leq j \leq 2 k .
\end{array}\right.
\end{aligned}
$$

To estimate error bounds induced by these new structures, we need the following Lemma. 
LEMMA 4.1. Let $\boldsymbol{\Psi}$ be a $2 k$ block vector of which the entries are $O\left(\Delta x^{2 k}\right)$. Then, for $k=1,2,3$, and for the spring-mass conditions (3.2), we have

$$
\begin{aligned}
& \boldsymbol{M}^{-1} \boldsymbol{\Psi}=\left(\begin{array}{c}
O\left(\Delta x^{2 k-1}\right) \\
O\left(\Delta x^{2-2}\right) \\
\vdots \\
O(\Delta x) \\
O(1)
\end{array}\right), \quad \boldsymbol{M}^{-1} \boldsymbol{\Delta}_{\mathbf{1}}=\left(\begin{array}{c}
O\left(\Delta x^{2 k-1}\right) \\
O\left(\Delta x^{2 k-2}\right) \\
\vdots \\
O(\Delta x) \\
O(1)
\end{array}\right), \\
& \boldsymbol{N}^{-1} \mathbf{\Psi}=\left(\begin{array}{c}
O\left(\Delta x^{2 k-1}\right) \\
O\left(\Delta x^{2 k-2}\right) \\
\vdots \\
O(\Delta x) \\
O(1)
\end{array}\right), \quad \boldsymbol{N}^{-1} \boldsymbol{\Delta}_{\mathbf{2}}=\left(\begin{array}{c}
O\left(\Delta x^{2 k-1}\right) \\
O\left(\Delta x^{2 k-2}\right) \\
\vdots \\
O(\Delta x) \\
O(1)
\end{array}\right) .
\end{aligned}
$$

Proof. From the expressions of $\boldsymbol{M}$ (4.8), $\boldsymbol{N}$ (4.14), $\boldsymbol{\Delta}_{1}$ (4.9), and $\boldsymbol{\Delta}_{2}$ (4.15), we explicitly verify (4.27) with MAPLE.

THEOREM 4.2 (Local truncation error). The coupling (4.17) of an $r$-th order twostage scheme and of the ESIM $k$ maintains an $r$-th order local error at the irregular points $(J-s+1, \ldots, J+s)$ if

$$
2 k-1 \geq r
$$

RESULT 6. To recover the time-stepping (2.6) in the limit case of an homogeneous medium, and to maintain the order of accuracy at the irregular points $(J-s+1, \ldots, J+$ s), relations (4.20) and (4.28) imply, for an $r$-th order two-stage scheme with a width $s$, this choice for $k$

$$
k=\max \left(s, r+1-\operatorname{trunc}\left(\frac{r+1}{2}\right)\right),
$$

where $\operatorname{trunc}(x)$ refers to the truncated value of $x$. Consequently to (4.29), LaxWendroff and finite volume with flux limiter schemes require $k \geq 2$, and WENO 5 requires $k \geq 3$.

We do not demonstrate the Theorem 4.2 , because the proof is very close to the one given in [23]. To see the key point, i.e. where the Lemma 4.1 arises, we propose the local truncation analysis in the case of the Lax-Wendroff scheme and for $k=2$. In this case, the irregular points are $x_{J}$ and $x_{J+1}$. As a consequence of (2.8) and (4.17), the time-stepping at $x_{J}$ is

$\frac{\boldsymbol{U}_{J}^{n+1}-\boldsymbol{U}_{J}^{n}}{\Delta t}+\frac{1}{2 \Delta x} \boldsymbol{A}_{0}\left(\boldsymbol{U}_{J+1}^{*}-\boldsymbol{U}_{J-1}^{n}\right)-\frac{1}{2}\left(\frac{c_{0}}{\Delta x}\right)^{2} \Delta t\left(\boldsymbol{U}_{J+1}^{*}-2 \boldsymbol{U}_{J}^{n}+\boldsymbol{U}_{J-1}^{n}\right)=\mathbf{0}$.

To compute the local truncation error $\mathcal{L}\left(x_{J}, t_{n}\right)$, each numerical value $\boldsymbol{U}_{i}^{n}$ that appears explicitly or implicitly in (4.30) is replaced by the exact value $\boldsymbol{U}\left(x_{i}, t_{n}\right)$. Consequently

$$
\mathcal{L}\left(x_{J}, t_{n}\right)=\mathcal{L}_{1}\left(x_{J}, t_{n}\right)+\mathcal{L}_{2}\left(x_{J}, t_{n}\right)+\mathcal{L}_{3}\left(x_{J}, t_{n}\right),
$$


with

$$
\begin{aligned}
& \mathcal{L}_{1}\left(x_{J}, t_{n}\right)=\frac{\boldsymbol{U}\left(x_{J}, t_{n+1}\right)-\boldsymbol{U}\left(x_{J}, t_{n}\right)}{\Delta t} \\
& \mathcal{L}_{2}\left(x_{J}, t_{n}\right)=\frac{1}{2 \Delta x} \boldsymbol{A}_{0}\left(\boldsymbol{U}^{*}\left(x_{J+1}, t_{n}\right)-\boldsymbol{U}\left(x_{J-1}, t_{n}\right)\right) \\
& \mathcal{L}_{3}\left(x_{J}, t_{n}\right)=-\frac{1}{2}\left(\frac{c_{0}}{\Delta x}\right)^{2} \Delta t\left(\boldsymbol{U}^{*}\left(x_{J+1}, t_{n}\right)-2 \boldsymbol{U}\left(x_{J}, t_{n}\right)+\boldsymbol{U}\left(x_{J-1}, t_{n}\right)\right) .
\end{aligned}
$$

The exact value $\boldsymbol{U}^{*}\left(x_{J+1}, t_{n}\right)$ corresponding to the modified value $\boldsymbol{U}_{J+1}^{*}$ is deduced from (4.10)

$$
\boldsymbol{U}^{*}\left(x_{J+1}, t_{n}\right)=\left(\boldsymbol{I}_{2}, \ldots, \frac{\left(x_{J+1}-\alpha\right)^{3}}{3 !} \boldsymbol{I}_{2}\right) \boldsymbol{M}^{-1}\left(\begin{array}{c}
\boldsymbol{U}\left(x_{J-1}, t_{n}\right) \\
\vdots \\
\boldsymbol{U}\left(x_{J+2}, t_{n}\right)
\end{array}\right) .
$$

Using (4.7) and the Lemma 4.1 for $k=2$ gives

$$
\begin{aligned}
\boldsymbol{U}^{*}\left(x_{J+1}, t_{n}\right)= & \left(\boldsymbol{I}_{2}, \ldots, \frac{\left(x_{J+1}-\alpha\right)^{3} \boldsymbol{I}_{2}}{3 !}\right) \boldsymbol{M}^{-1}
\end{aligned}\left\{\begin{array}{c}
\boldsymbol{M}\left(\begin{array}{c}
\boldsymbol{U}\left(\alpha^{-}, t_{n}\right) \\
\vdots \\
\partial^{3} \\
\frac{\boldsymbol{U}^{3}}{}\left(\alpha^{-}, t_{n}\right)
\end{array}\right) \\
+\boldsymbol{\Delta}_{1}+\left(\begin{array}{c}
\boldsymbol{O}\left(\Delta x^{4}\right) \\
\vdots \\
\boldsymbol{O}\left(\Delta x^{4}\right)
\end{array}\right) \\
=\sum_{m=0}^{3} \frac{\left(x_{J+1}-\alpha\right)^{m}}{m !} \frac{\partial^{k}}{\partial x^{m}} \boldsymbol{U}\left(\alpha^{-}, t_{n}\right)+\boldsymbol{O}\left(\Delta x^{3}\right) .
\end{array}\right\}
$$

$\mathcal{L}_{1}$ is deduced from Taylor expansions around $\left(\alpha^{-}, t_{n}\right)$ and from $(2.5)$

$$
\begin{aligned}
\mathcal{L}_{1}\left(x_{J}, t_{n}\right) & =\frac{\partial}{\partial t} \boldsymbol{U}\left(x_{J}, t_{n}\right)+\frac{\Delta t}{2} \frac{\partial^{2}}{\partial t^{2}} \boldsymbol{U}\left(x_{J}, t_{n}\right)+\boldsymbol{O}\left(\Delta t^{2}\right) \\
& =-\boldsymbol{A}_{0} \frac{\partial}{\partial x} \boldsymbol{U}\left(x_{J}, t_{n}\right)+c_{0}^{2} \frac{\Delta t}{2} \frac{\partial^{2}}{\partial x^{2}} \boldsymbol{U}\left(x_{J}, t_{n}\right)+\boldsymbol{O}\left(\Delta t^{2}\right) \\
& =-\boldsymbol{A}_{0} \frac{\partial}{\partial x} \boldsymbol{U}\left(\alpha^{-}, t_{n}\right)+\left(c_{0}^{2} \frac{\Delta t}{2}-\left(x_{J}-\alpha\right) \boldsymbol{A}_{0}\right) \frac{\partial^{2}}{\partial x^{2}} \boldsymbol{U}\left(\alpha^{-}, t_{n}\right)+\boldsymbol{O}\left(\Delta x^{2}\right) .
\end{aligned}
$$

In the last line of (4.34), we use the fact that an $O\left(\Delta t^{2}\right)$ function is also an $O\left(\Delta x^{2}\right)$ function because the CFL number is kept constant. $\mathcal{L}_{2}$ and $\mathcal{L}_{3}$ are deduced from second-order Taylor expansions of $\boldsymbol{U}\left(x_{J-1}, t_{n}\right)$ and $\boldsymbol{U}\left(x_{J}, t_{n}\right)$ at $\alpha^{-}$, and from (4.33)

$$
\begin{aligned}
\mathcal{L}_{2}\left(x_{J}, t_{n}\right) & =\boldsymbol{A}_{0} \frac{\partial}{\partial x} \boldsymbol{U}\left(\alpha^{-}, t_{n}\right)+\left(x_{J}-\alpha\right) \boldsymbol{A}_{0} \frac{\partial^{2}}{\partial x^{2}} \boldsymbol{U}\left(\alpha^{-}, t_{n}\right)+\boldsymbol{O}\left(\Delta x^{2}\right) \\
\mathcal{L}_{3}\left(x_{J}, t_{n}\right) & =-c_{0}^{2} \frac{\Delta t}{2} \frac{\partial^{2}}{\partial x^{2}} \boldsymbol{U}\left(\alpha^{-}, t_{n}\right)+O\left(\Delta x^{2}\right) .
\end{aligned}
$$


Summing $\mathcal{L}_{1}, \mathcal{L}_{2}$, and $\mathcal{L}_{3}$ allows one to conclude

$$
\mathcal{L}\left(x_{J}, t_{n}\right)=\boldsymbol{O}\left(\Delta x^{2}\right),
$$

which is the local truncation error of the Lax-Wendroff scheme at regular points. A similar analysis can be done at $x_{J+1}$.

\section{Numerical experiments.}

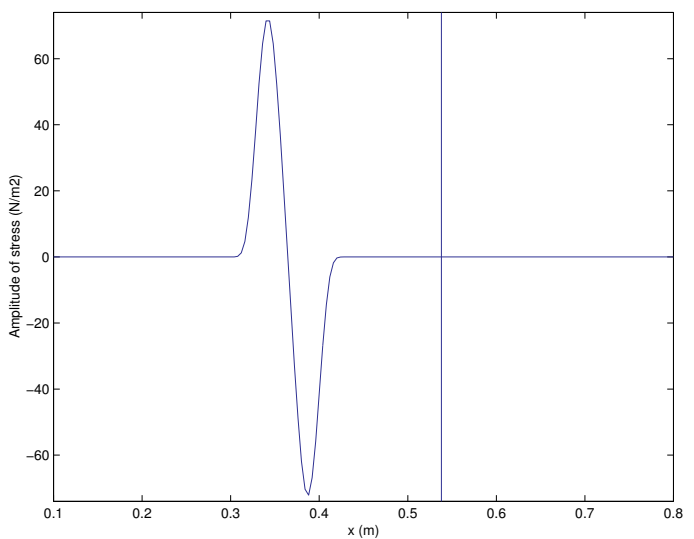

FIG. 5.1. Initial values of the elastic stress $\sigma$ at $t=t_{0}=1.610^{-4} \mathrm{~s}$.

5.1. One interface between identical media. Consider a $L=1 \mathrm{~m}$ elastic domain with an imperfect interface at $\alpha=0.533 \mathrm{~m}$. The parameters are

$$
\left\{\begin{array}{l}
\rho_{0}=\rho_{1}=1200 \mathrm{~kg} / \mathrm{m}^{3}, \\
c_{0}=c_{1}=2800 \mathrm{~m} / \mathrm{s}, \\
K=2.25 .10^{11} \mathrm{~kg} / \mathrm{s}^{2}, \\
M=10 \mathrm{~kg} / \mathrm{m}^{2} .
\end{array}\right.
$$

Since there are no impedance contrasts, the reflected wave is only due to the springmass conditions. The values of $K$ and $M$ correspond e.g. to the case of a glue layer with the physical properties of water $\rho_{2}=1000 \mathrm{~kg} / \mathrm{m}^{3}, c_{2}=1500 \mathrm{~m} / \mathrm{s}$, and with a thickness $h=0.01 \mathrm{~m}(\mathrm{~A} .4)$. The initial data is given by a right-going wave in medium 0

$$
\boldsymbol{U}_{0}(x)=f\left(t_{0}-\frac{x}{c_{0}}\right)\left(\begin{array}{c}
-\frac{1}{c_{0}} \\
\rho_{0}
\end{array}\right) .
$$

The function $f$ is a $C^{5}$ spatially-bounded sinusoid

$$
f(\xi)=\left\{\begin{array}{l}
\sum_{k=1}^{q} a_{k} \sin \left(\beta_{k} \omega_{c} \xi\right) \quad \text { if } 0<\xi<\frac{1}{f_{c}} \\
0 \text { else, }
\end{array}\right.
$$


(a)

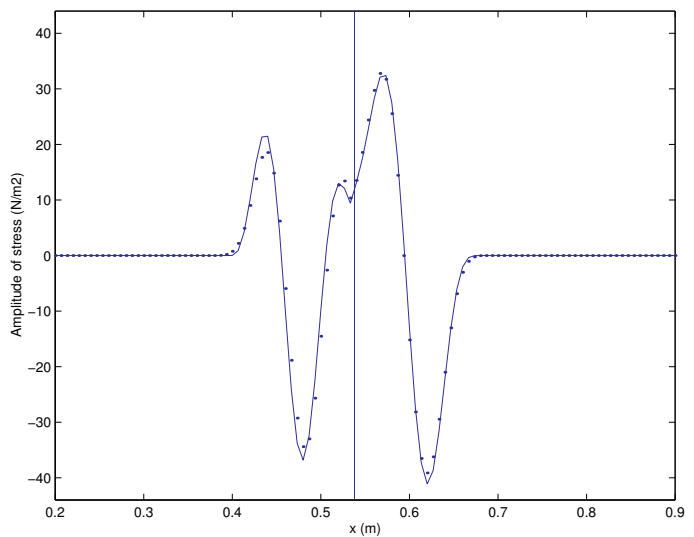

(c)

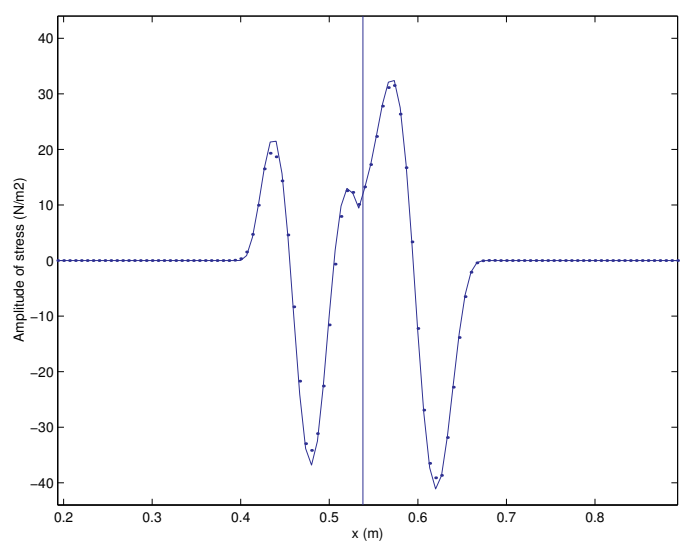

(e)

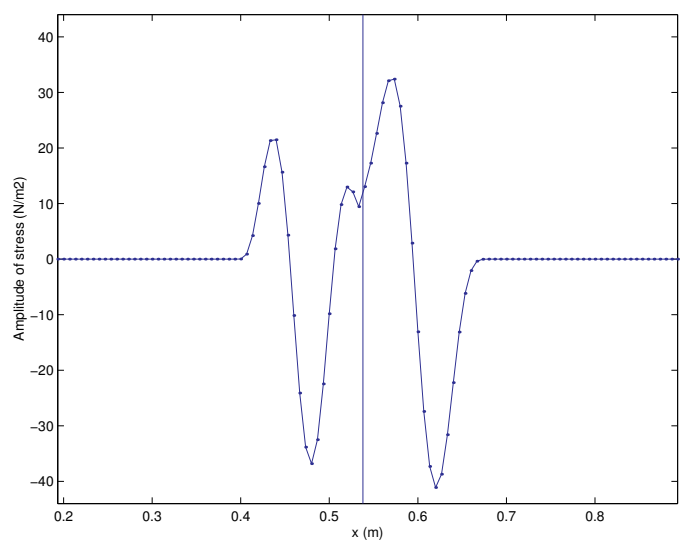

(b)

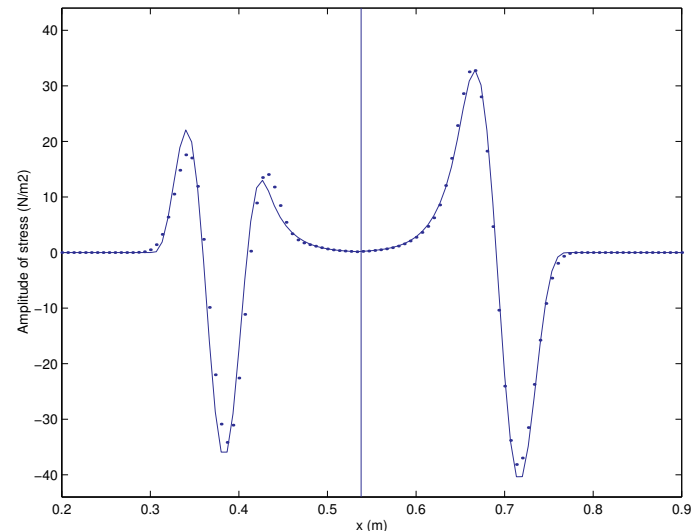

(d)

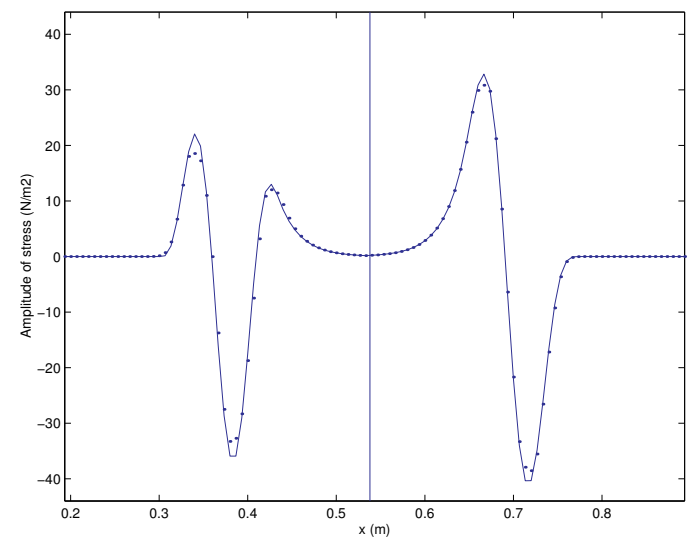

(f)

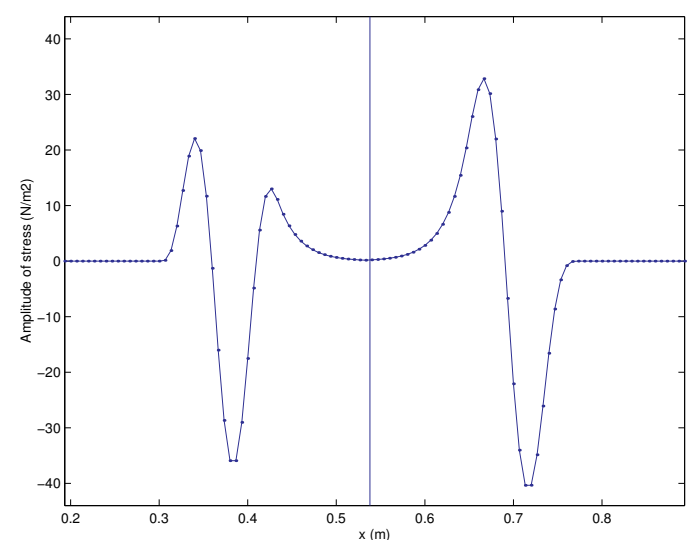

FIG. 5.2. Exact values (solid line) and numerical values (dotted line) of $\sigma$ for identical properties on both sides of an imperfect interface. Lax-Wendroff + ESIM 2 ( $a$-b), finite volume with flux limiter + ESIM 2 (c-d), WENO $5+$ ESIM $3(e-f)$. Snapshots at $t_{1}=2.610^{-4} \mathrm{~s}$ (left row) and at $t_{2}=2.910^{-4} \mathrm{~s}$ (right row). 
with $\beta_{k}=2^{k-1}, \omega_{c}=2 \pi f_{c}$; the coefficients $a_{k}$ are: $a_{1}=1, a_{2}=-21 / 32, a_{3}=63 / 768$, $a_{4}=-1 / 512$. The central frequency is $f_{c}=210^{4} \mathrm{~Hz}$.

The numerical experiments shown in Fig 5.2 are performed with $\mathrm{N}=150$ grid points, which is approximately 20 points in the wavelength $\lambda_{c}=c_{0} / f_{c}$, and with $\mathrm{CFL}=0.9$ (2.7). The initial time is $t_{0}=1.610^{-4} \mathrm{~s}$. Initial values of the elastic stress $\sigma$ are shown in Fig. 5.1.

\begin{tabular}{|c|c|c|c|c|c|}
\hline Method & $\mathrm{N}$ & $L_{\infty}$ error & $L_{\infty}$ order & $L_{1}$ error & $L_{1}$ order \\
\hline $\begin{array}{c}\text { Lax-Wendroff } \\
+ \\
\text { ESIM } 2\end{array}$ & $\begin{array}{c}100 \\
200 \\
400 \\
800 \\
1600 \\
3200\end{array}$ & $\begin{array}{l}1.12 \mathrm{e} 01 \\
4.03 \mathrm{e} 00 \\
9.75 \mathrm{e}-1 \\
2.45 \mathrm{e}-1 \\
5.95 \mathrm{e}-2 \\
1.48 \mathrm{e}-2\end{array}$ & $\begin{array}{c}- \\
1.47 \\
2.04 \\
1.99 \\
2.04 \\
2.01\end{array}$ & $\begin{array}{c}1.43 \mathrm{e} 00 \\
4.60 \mathrm{e}-1 \\
1.19 \mathrm{e}-1 \\
3.04 \mathrm{e}-2 \\
7.57 \mathrm{e}-3 \\
1.89 \mathrm{e}-3\end{array}$ & $\begin{array}{c}- \\
1.61 \\
1.96 \\
1.97 \\
2.00 \\
2.00\end{array}$ \\
\hline $\begin{array}{c}\text { Finite volume } \\
+ \\
\text { ESIM } 2\end{array}$ & $\begin{array}{c}100 \\
200 \\
400 \\
800 \\
1600 \\
3200\end{array}$ & $\begin{array}{l}6.05 \mathrm{e} 00 \\
2.00 \mathrm{e} 00 \\
6.77 \mathrm{e}-1 \\
2.35 \mathrm{e}-2 \\
7.92 \mathrm{e}-3 \\
2.63 \mathrm{e}-2\end{array}$ & $\begin{array}{c}- \\
1.59 \\
1.56 \\
1.52 \\
1.56 \\
1.59\end{array}$ & $\begin{array}{l}5.05 \mathrm{e}-1 \\
1.31 \mathrm{e}-1 \\
3.45 \mathrm{e}-2 \\
9.76 \mathrm{e}-3 \\
2.59 \mathrm{e}-3 \\
6.39 \mathrm{e}-4\end{array}$ & $\begin{array}{c}- \\
1.94 \\
1.92 \\
1.82 \\
1.91 \\
2.02\end{array}$ \\
\hline $\begin{array}{c}\text { WENO } 5 \\
+ \\
\text { ESIM } 3\end{array}$ & $\begin{array}{c}100 \\
200 \\
400 \\
800 \\
1600 \\
3200\end{array}$ & $\begin{array}{l}6.05 \mathrm{e} 00 \\
2.08 \mathrm{e}-1 \\
6.60 \mathrm{e}-3 \\
2.21 \mathrm{e}-4 \\
7.39 \mathrm{e}-6 \\
2.45 \mathrm{e}-7\end{array}$ & $\begin{array}{c}- \\
4.85 \\
4.98 \\
4.89 \\
4.90 \\
4.91\end{array}$ & $\begin{array}{l}3.20 \mathrm{e} 00 \\
1.09 \mathrm{e}-1 \\
3.71 \mathrm{e}-3 \\
1.19 \mathrm{e}-4 \\
4.06 \mathrm{e}-6 \\
1.34 \mathrm{e}-7\end{array}$ & $\begin{array}{c}- \\
4.86 \\
4.88 \\
4.95 \\
4.88 \\
4.92\end{array}$ \\
\hline
\end{tabular}

TABLE 5.1

Errors and orders of accuracy.

Figure 5.2 shows the exact values and the numerical values of the elastic stress $\sigma$ at $t_{1}=2.610^{-4} \mathrm{~s}$ (left row) and at $t_{2}=2.910^{-4} \mathrm{~s}$ (right row). The analytic solution is detailed in Appendix B. There is an excellent agreement between the theoretical values and the numerical values, and we can observe the features of each scheme, that is the numerical dispersion of the Lax-Wendroff scheme (a-b) and the numerical diffusion of the finite volume scheme with flux limiter (c-d); at this scale, we can not observe differences between the theoretical values and the numerical values computed with WENO 5 (e-f).

Table 5.1 shows the errors in norms $L_{\infty}$ and $L_{1}$ at $t_{2}=2.910^{-4} \mathrm{~s}$. For WENO 5 , the time step has been adjusted to $\Delta t \sim(\Delta x)^{5 / 4}$ so that the fourth-order integration in time is effectively fifth-order. All computing is done in double precision on a Pentium PC. As expected, the order of accuracy of all schemes is maintained with 
one imperfect interface.

(a)

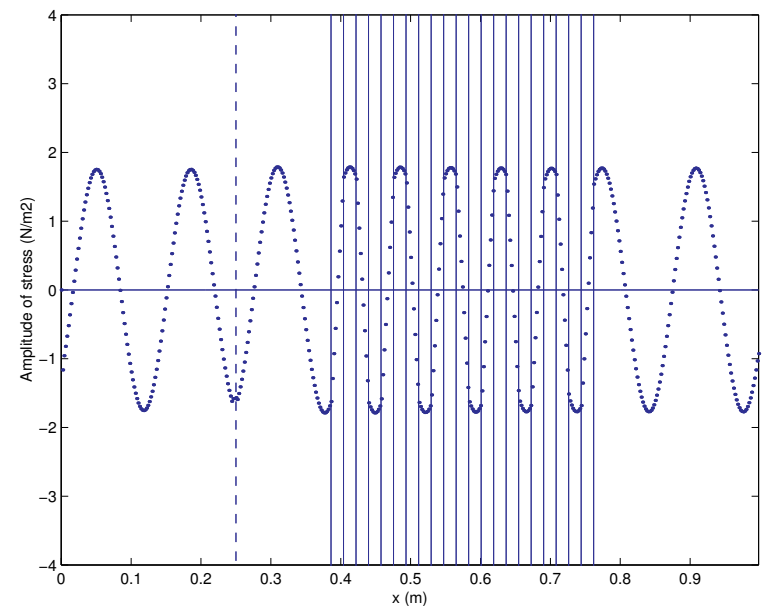

(b)

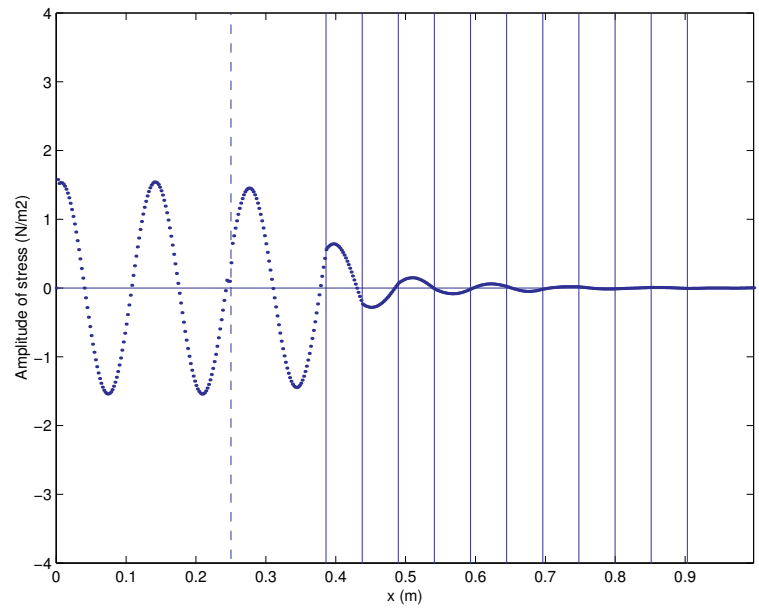

FIG. 5.3. Waves transmitted through a medium containing equally spaced imperfect interfaces, with (a) $h=h_{0}$, and (b) $h=h_{1}$. The vertical dotted line denotes the position of the source.

5.2. Periodic medium. As a second test, we consider the wave propagation across a bounded set of imperfect interfaces equally spaced of $h$, with the physical properties of the previous example. The wave propagation in such a medium has been extensively studied for the spring conditions (i.e. $M=0$ ) and using Floquet's theory [20]. The derivation of the dispersion relation for Floquet waves with the spring-mass conditions is beyond the scope of the present paper. A theoretical analysis shows that for $h=h_{0}=1.7610^{-2} \mathrm{~m}$, the Floquet wave number is real, hence waves propagate without any loss. On the contrary, for $h=h_{1}=5.1710^{-2} \mathrm{~m}$, the Floquet wave number is complex. This results in evanescent waves that decrease exponentially away from sources. This stop-pass behavior is well-known in electromagnetics and geophysics. It can be applied to construct an acoustic filter of which properties can be altered by changing the spacing between interfaces. 


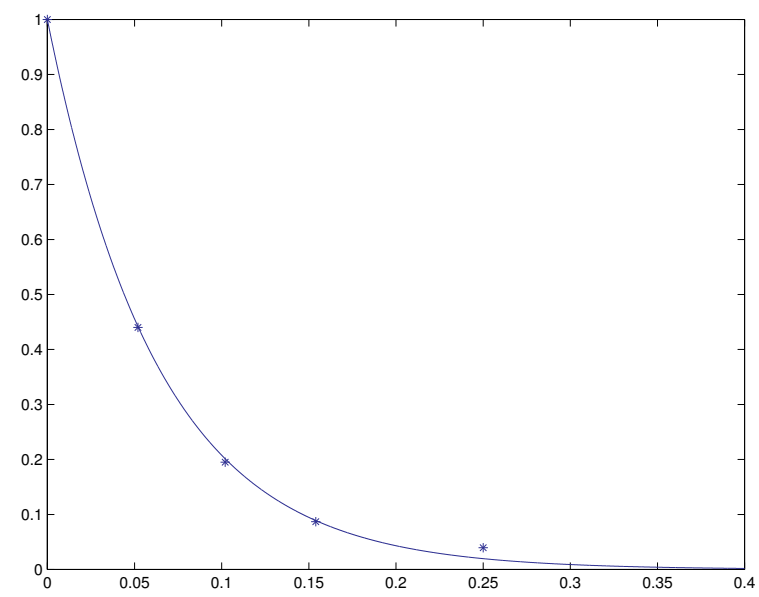

FIG. 5.4. Theoretical values (solid line) and numerical values (*) of the attenuation in the periodic medium.

The numerical simulations are performed with 250 grid points, with the LaxWendroff scheme coupled to the ESIM 2. A sinusoidal source is imposed on the left of the set of interfaces (dotted vertical line in Fig. 5.3), until a steady-state is reached. The stop-pass behavior is clearly observed in Fig. 5.3. Figure 5.4 shows the theoretical attenuation (solid line) and the measured attenuation $\left(^{*}\right)$ of the waves. Once again, the agreement between exact and numerical values is excellent.

On this example, the additional computational cost required by the interface method is lower than $1 \%$, hence it is negligible compared with the time-stepping of the scheme. Note also that no instabilities are observed, despite the multiple reflections in the periodic medium (unlike interface problems studied by [9]).

5.3. One interface between different media. As a third example, we consider one imperfect interface between different media, with discontinuous coefficients

$$
(\rho, c)= \begin{cases}\rho_{0}=1200 \mathrm{~kg} / \mathrm{m}^{3}, & c_{0}=2800 \mathrm{~m} / \mathrm{s}, \\ \rho_{1}=2700 \mathrm{~kg} / \mathrm{m}^{3}, & c_{1}=6500 \mathrm{~m} / \mathrm{s} .\end{cases}
$$

These physical parameters correspond respectively to Plexiglass and to aluminium. We propose a parametric study for various values of the stiffness: $K=10^{13} \mathrm{~kg} / \mathrm{s}^{2}$, $K=710^{11} \mathrm{~kg} / \mathrm{s}^{2}, K=2.2510^{11} \mathrm{~kg} / \mathrm{s}^{2}, K=710^{10} \mathrm{~kg} / \mathrm{s}^{2}$. The inertial effects are not considered: $M=0$. The computations are performed with the finite volume scheme coupled with the ESIM 2. The values of $\alpha, \mathrm{N}, f_{c}$ and $t_{0}$ are the same than in section 5.1.

Figure 5.5 shows the exact values (solid line) and the numerical values (dotted line) of $\sigma$ at $t_{1}=2.410^{-4} \mathrm{~s}$ (left row) and at $t_{2}=2.510^{-4} \mathrm{~s}$ (right row), after respectively 140 and 180 time steps. Measures of convergence have been performed (not shown here) confirm the second-order accuracy. Note that we can not distinguish the simulation (a-b) from simulations with a perfect contact $(K \rightarrow+\infty)$.

Besides its numerical interest, this test is enlightening from a practical point of view. We observe a clear difference between the fields for a perfect contact (a-b) and for thin glue layers (c-h). Many other simulations have been investigated, especially 
(a)

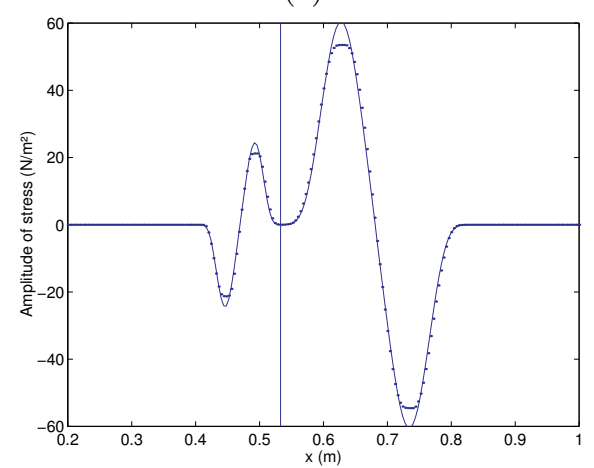

(c)

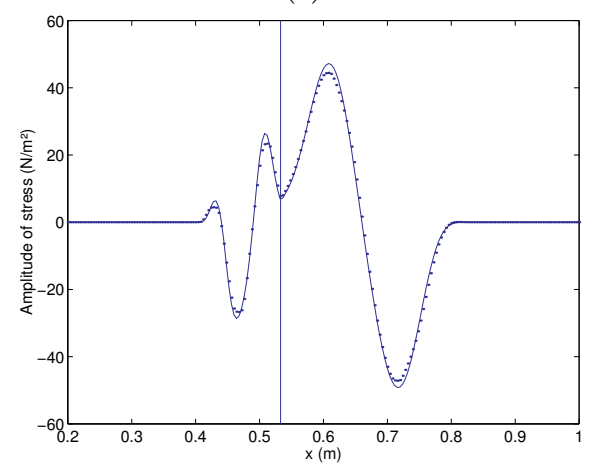

(e)

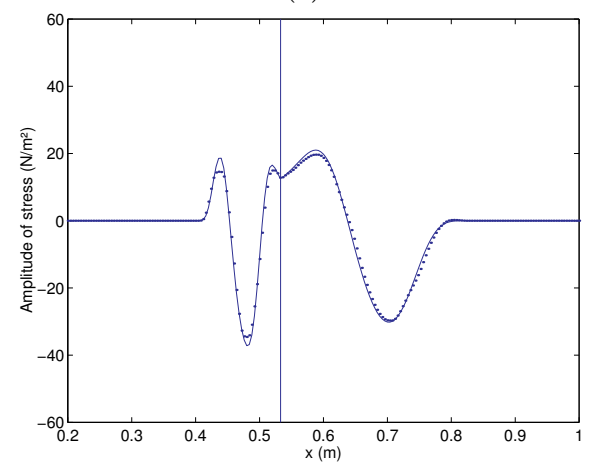

$(\mathrm{g})$

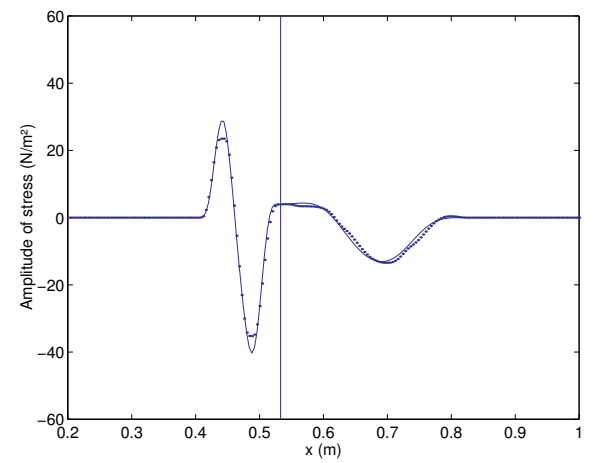

(b)

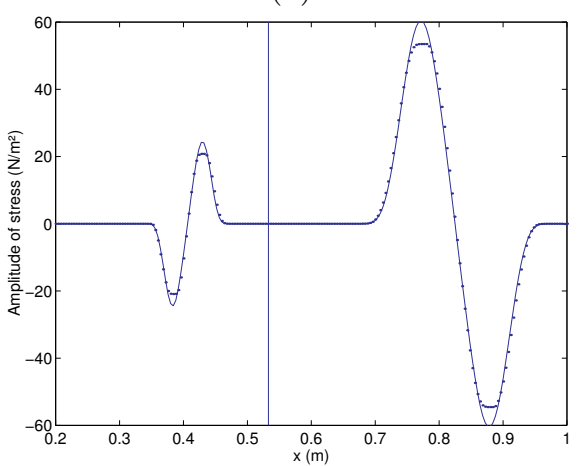

(d)

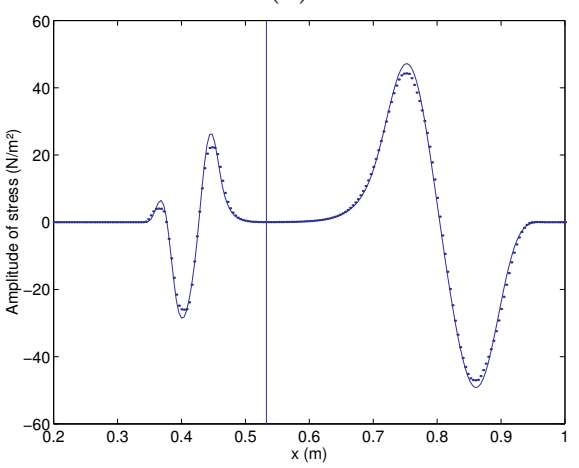

(f)

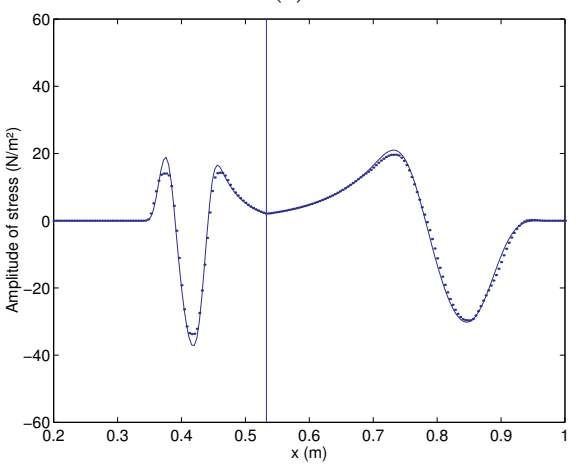

(h)

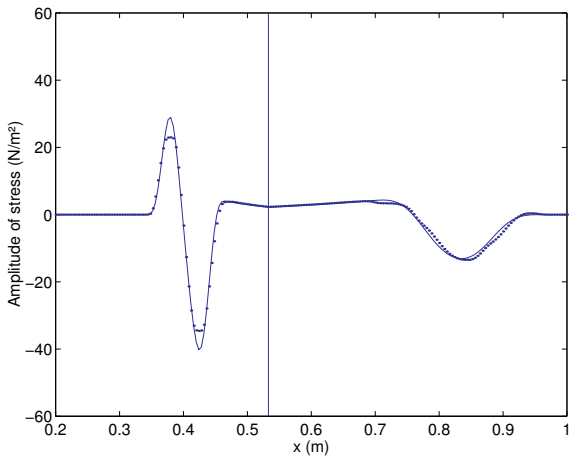

FIG. 5.5. Discontinuous coefficients at $t_{1}=2.610^{-4} \mathrm{~s}$ (left row), $t_{2}=2.910^{-4} \mathrm{~s}$ (right row): $K=10^{13} \mathrm{~kg} / \mathrm{s}^{2}(a-b), K=710^{11} \mathrm{~kg} / \mathrm{s}^{2}(c-d), K=2.2510^{11} \mathrm{~kg} / \mathrm{s}^{2}(e-f), K=710^{10} \mathrm{~kg} / \mathrm{s}^{2}(g-h)$. 
for very small values of $K$ (like $K=10^{-3} \mathrm{~kg} / \mathrm{s}^{2}$ ). It results in stable schemes, despite the huge $1 / K$ factor in the matrices $\boldsymbol{D}$ (3.6), leading to totally reflected wave.

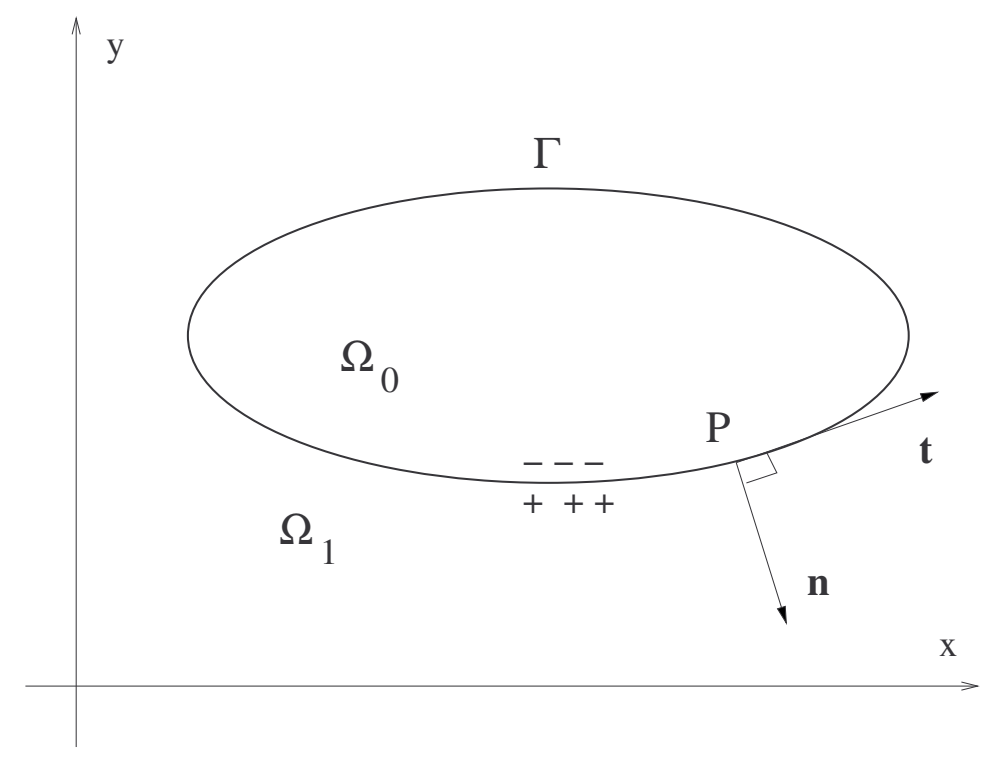

FIG. 5.6. Interface $\Gamma$ between $\Omega_{0}$ and $\Omega_{1}$.

5.4. Two-dimensional example. Lastly, we propose a $2 \mathrm{D}$ application of the spring-mass conditions. The goal of this example is only to show that those conditions can be treated by the ESIM. Technical details therefore are systematically omitted, and they will be treated in future works.

Consider a sufficiently smooth interface $\Gamma$ dividing $\mathbb{R}^{2}$ into two subdomains $\Omega_{0}$ and $\Omega_{1}$ (Fig. 5.6). At any point $P$ of $\Gamma$, we define a normal unit vector $\boldsymbol{n}$ and a tangential unit vector $\boldsymbol{t}$. Denoting by $\boldsymbol{u}, \boldsymbol{v}$ and $\boldsymbol{\sigma}$ respectively the elastic displacement, the elastic velocity and the elastic stress tensor, we write

$$
\begin{array}{|l|l|l}
u_{N}=\boldsymbol{u} . \boldsymbol{n}, & v_{N}=\boldsymbol{v} . \boldsymbol{n}, & \sigma_{N}=(\boldsymbol{\sigma} \cdot \boldsymbol{n}) . \boldsymbol{n}, \\
u_{T}=\boldsymbol{u . t} & v_{T}=\boldsymbol{v . t} & \sigma_{T}=(\boldsymbol{\sigma} \cdot \boldsymbol{n}) . \boldsymbol{t} .
\end{array}
$$

The limit values of a function $f(x, y, t)$ at $P$ on both sides of $\Gamma$ are written

$$
f\left(P^{+}, t\right)=\lim _{M \rightarrow P, M \in \Omega_{1}} f(M, t), \quad f\left(P^{-}, t\right)=\lim _{M \rightarrow P, M \in \Omega_{0}} f(M, t) .
$$

We denote by

$$
[f(P, t)]=f\left(P^{+}, t\right)-f\left(P^{-}, t\right)
$$

the jump of $f$ across $\Gamma$ in $P$, from $\Omega_{0}$ to $\Omega_{1}$. Given four constants $K_{N}>0, K_{T}>0$, $M_{N} \geq 0, M_{T} \geq 0$, respectively called the normal stiffness, the tangential stiffness, the normal mass and the tangential mass of the interface, the two-dimensional spring-mass 
conditions are

$$
\begin{aligned}
& {\left[u_{N}(P, t)\right]=\frac{1}{K_{N}} \sigma_{N}\left(P^{-}, t\right),} \\
& {\left[u_{T}(P, t)\right]=\frac{1}{K_{T}} \sigma_{T}\left(P^{-}, t\right),} \\
& {\left[\sigma_{N}(P, t)\right]=M_{N} \frac{\partial^{2}}{\partial t^{2}} u_{N}\left(P^{-}, t\right),} \\
& {\left[\sigma_{T}(P, t)\right]=M_{T} \frac{\partial^{2}}{\partial t^{2}} u_{T}\left(P^{-}, t\right) .}
\end{aligned}
$$

These conditions generalize the $1 \mathrm{D}$ conditions $(3.2)$.

As an example, we consider a $L_{x} \times L_{y}=0.4 \times 0.4 \mathrm{~m}^{2}$ elastic domain with an imperfect contact along a horizontal interface at $y=0.113 \mathrm{~m}$. The physical parameters are

$$
\left\{\begin{array}{l}
\rho_{0}=\rho_{1}=1200 \mathrm{~kg} / \mathrm{m}^{3} \\
c_{P 0}=c_{P 1}=2800 \mathrm{~m} / \mathrm{s} \\
c_{S 0}=c_{S 1}=1300 \mathrm{~m} / \mathrm{s} \\
K_{N}=910^{11} \mathrm{~kg} / \mathrm{s}^{2} \\
K_{T}=10^{11} \mathrm{~kg} / \mathrm{s}^{2} \\
M_{N}=M_{T}=0 \mathrm{~kg} / \mathrm{m}^{2}
\end{array}\right.
$$

$c_{P i}$ and $c_{S i}(i=0,1)$ denote respectively the celerities of $\mathrm{P}$-waves and S-waves. See [4] for properties of those waves. Like in the first 1D example, we choose identical densities and celerities on both sides of the interface in order to visualize clearly the effect of spring conditions: since there are no impedance contrasts, reflections and conversions of waves are only induced by the stiffnesses. The interface considered here is plane and horizontal for esthetic purpose; however, the ESIM can treat without difficulty arbitrary-shaped smooth interfaces that cut the uniform cartesian grid. The parameters (5.9) describe the following realistic configuration: two blocks of Plexiglass linked together by a glue layer.

Numerical experiments are performed with $N_{x} \times N_{y}=400 \times 400$ grid points, with $\mathrm{CFL}=0.69$. The computation is initialized by a cylindrical wave centered at $(0.2 \mathrm{~m}$, $0.2 \mathrm{~m}$ ). For time-stepping, we use a finite-volume scheme with flux limiter, the Wave Propagation Algorithm developed by LeVeque [14, 16]. This scheme is coupled with the ESIM to take into account the conditions at the interface. Concerning the palettes of colours, we use a green-red palette for P-waves, and a magenta-yellow palette for S-waves. The distinction between those waves lies on numerical estimations of $\boldsymbol{\nabla} \wedge \boldsymbol{v}$ and of $\boldsymbol{\nabla} \cdot \boldsymbol{v}$.

Figure 5.7 shows snapshots of $v_{1}$ (left column) and $v_{2}$ (right column), respectively the horizontal component of the elastic velocity and the vertical component of the elastic velocity, at various instants: at the initialization (a-b), after 100 time 
(a)

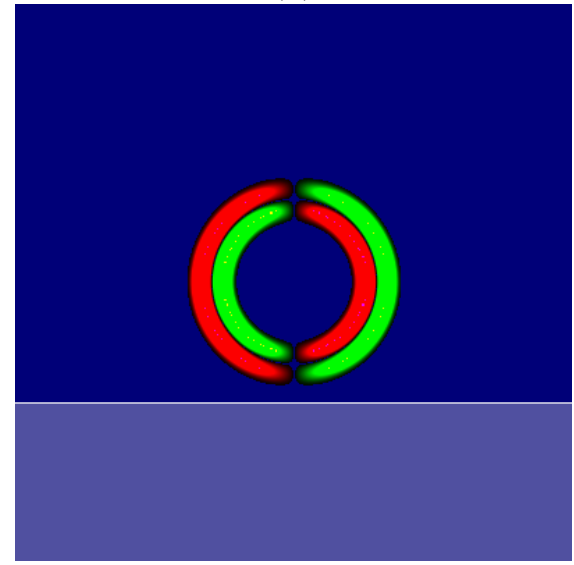

(c)

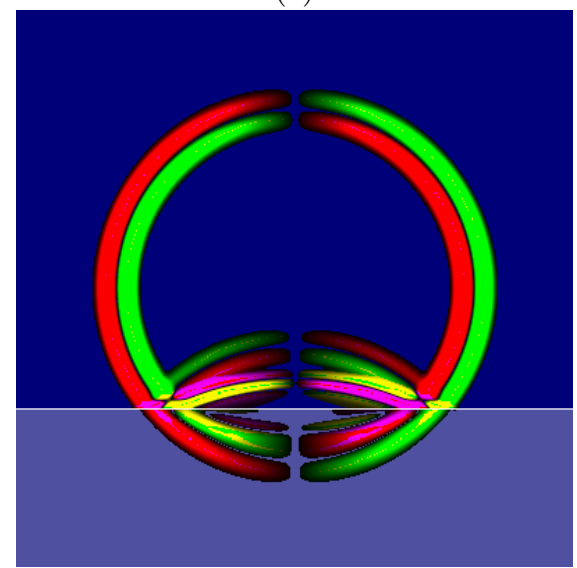

(e)

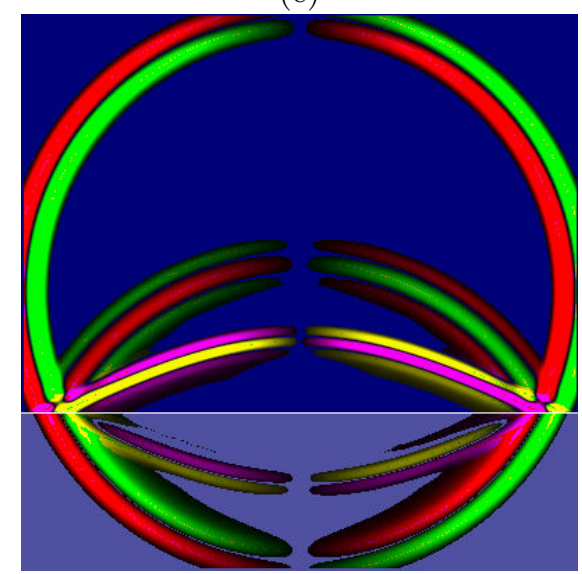

FIG. 5.7. Snapshots of $v_{1}(a-c-e)$ and $v_{2}(b-d-f)$. Green-red: P-waves, yellow-magenta: S-waves (b)

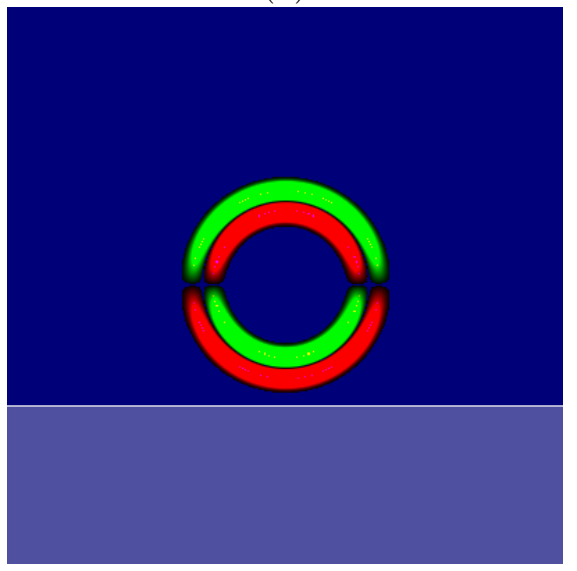

(d)

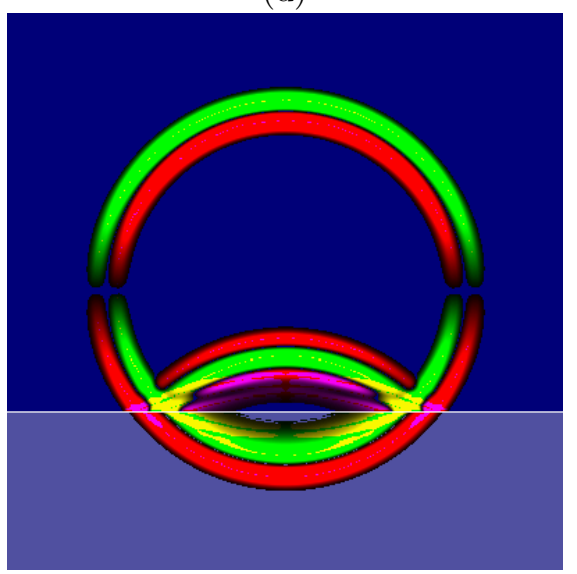

(f)

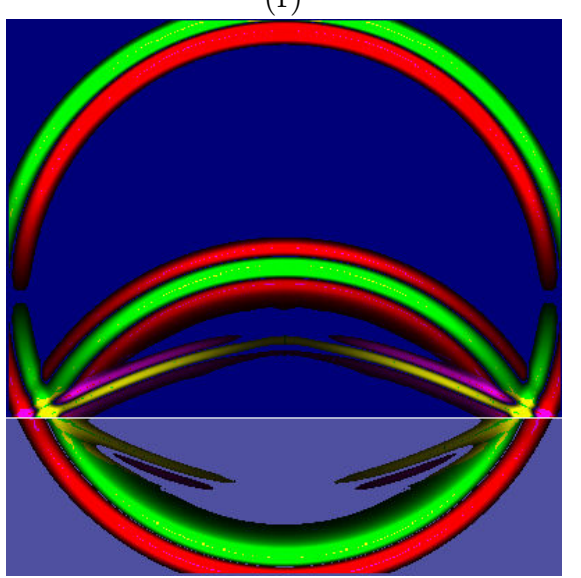


steps (c-d), after 200 time steps (e-f). Interesting wave phenomenons are observed. The incident $\mathrm{P}$-wave is reflected and transmitted at the interface. The reflected and transmitted P-waves are followed by slower S-waves. Since there are no impedance contrasts, the S-waves are only induced by $K_{N}$ and $K_{T}$. Lastly, we observe that a wake follows $\mathrm{P}$ and $\mathrm{S}$ reflected and transmitted waves: like in the $1 \mathrm{D}$ case, those waves are not spatially bounded, because of the frequential dependance induced by $K_{N}$ and $K_{T}$.

6. Conclusion. In this paper, we have described a procedure to incorporate the spring-mass conditions in explicit two-step finite-difference schemes issued from computational fluid dynamics. For that purpose, we couple the schemes with the Explicit Simplified Interface Method (ESIM) [23]. The same procedure can be applied to other schemes with minor changes. A local truncation analysis and numerical experiments have shown that properties of schemes are maintained with imperfect interfaces. Lastly, the additional computational cost induced by the ESIM is negligible.

In the future, we want to investigate deeper 2D and 3D cases of wave propagation across imperfect contacts, like the propagation of interface waves along fractures [11]. Since the properties of those waves depend on the nature of the interface, they give insight on degrees of contact between solids, leading to applications in non-destructive evaluations of materials and in geophysics.

The extension of the ESIM to more complicated jump conditions is another challenging project. We think to nonlinear jump conditions, depending on $t$ and on the solution itself, that describe realistic contacts in solid mechanics.

Appendix A. Derivation of the spring-mass conditions. In this appendix,

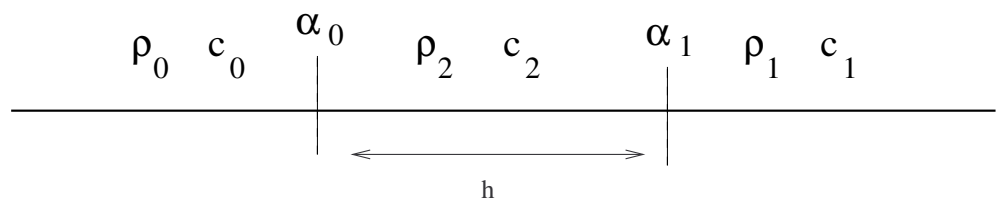

FIG. A.1. 1D elastic medium with two perfect interfaces.

we deduce the spring-mass conditions from the propagation of elastic waves in a threelayered medium with two perfect interface at $\alpha_{0}$ and $\alpha_{1}$ (as shown in Fig. A.1). We set $h=\alpha_{1}-\alpha_{0}$; the density and the celerity of elastic waves in medium $\Omega_{2}$ are respectively $\rho_{2}$ and $c_{2}$. Since the contact is perfect at $\alpha_{1}$, we get

$$
\boldsymbol{U}\left(\alpha_{1}^{+}, t\right)=\boldsymbol{U}\left(\alpha_{1}^{-}, t\right)
$$

A Taylor expansion of $\boldsymbol{U}\left(\alpha_{1}^{-}, t\right)$ at $\alpha_{0}^{+}$leads to

$$
\boldsymbol{U}\left(\alpha_{1}^{-}, t\right)=\boldsymbol{U}\left(\alpha_{0}^{+}, t\right)+h \frac{\partial}{\partial x} \boldsymbol{U}\left(\alpha_{0}^{+}, t\right)+\boldsymbol{O}\left(h^{2}\right)
$$


Lastly, the perfect conditions at $\alpha_{0}$ imply

$$
\begin{aligned}
& \boldsymbol{U}\left(\alpha_{0}^{+}, t\right)=\boldsymbol{U}\left(\alpha_{0}^{-}, t\right), \\
& \frac{\partial}{\partial x} \boldsymbol{U}\left(\alpha_{0}^{+}, t\right)=\operatorname{diag}\left(\frac{\rho_{0} c_{0}^{2}}{\rho_{2} c_{2}^{2}}, \frac{\rho_{2}}{\rho_{0}}\right) \frac{\partial}{\partial x} \boldsymbol{U}\left(\alpha_{0}^{-}, t\right) .
\end{aligned}
$$

Setting

$$
K=\frac{\rho_{2} c_{2}^{2}}{h}, \quad M=\rho_{2} h,
$$

we deduce from (A.1), (A.2) and (A.3)

$$
\begin{aligned}
& v\left(\alpha_{1}^{+}, t\right)=v\left(\alpha_{0}^{-}, t\right)+\frac{\rho_{0} c_{0}^{2}}{K} \frac{\partial}{\partial x} v\left(\alpha_{0}^{-}, t\right)+O\left(h^{2}\right) \\
& \sigma\left(\alpha_{1}^{+}, t\right)=\sigma\left(\alpha_{0}^{-}, t\right)+\frac{M}{\rho_{0}} \frac{\partial}{\partial x} \sigma\left(\alpha_{0}^{-}, t\right)+O\left(h^{2}\right) .
\end{aligned}
$$

For $h \rightarrow 0$, we recover the conditions (3.4) at $\alpha \in\left[\alpha_{0}, \alpha_{1}\right]$.

Appendix B. Time-domain solution with spring-mass conditions. We seek $v(x, t)$ and $\sigma(x, t)$ for one imperfect interface with the spring-mass conditions (3.2) (see Fig. 1.1). The computation is based on

1. a Fourier transform in time of the incident wave (5.3),

2. the determination of reflected and transmitted harmonic waves,

3. an inverse Fourier transform to obtain wave fields in the time domain.

First, we determine reflection and transmission coefficients (point 2). To do so, we consider incident, reflected, and transmitted harmonic waves, with amplitudes $A_{I}$, $A_{R}$, and $A_{T}$. Fourier transforms in time of the elastic potential $\Phi(x, t)$, of $u(x, t)$, and of $\sigma(x, t)$ are written in medium 0

$$
\begin{aligned}
& \hat{\Phi}(x, \omega)=A_{I} e^{-i k_{0} x}+A_{R} e^{i k_{0} x} \\
& \hat{u}(x, \omega)=-i k_{0} A_{I} e^{-i k_{0} x}+i k_{0} A_{R} e^{i k_{0} x} \\
& \hat{\sigma}(x, \omega)=-\rho_{0} \omega^{2} A_{I} e^{-i k_{0} x}-\rho_{0} \omega^{2} A_{R} e^{i k_{0} x}
\end{aligned}
$$

and in medium 1

$$
\begin{aligned}
\hat{\Phi}(x, \omega) & =A_{T} e^{-i k_{1} x} \\
\hat{u}(x, \omega) & =-i k_{1} A_{T} e^{-i k_{1} x} \\
\hat{\sigma}(x, \omega) & =-\rho_{1} \omega^{2} A_{T} e^{-i k_{1} x},
\end{aligned}
$$

where $k_{j}$ is the wavenumber in medium $j$, and $\omega$ is the angular frequency. The time dependance $e^{i \omega t}$ has been removed. Substituting (B.1) and (B.2) in the spring-mass conditions (3.2) leads to a system of equation of which solutions are $A_{R}$ and $A_{T}$. We deduce the reflection coefficient $R$ and the transmission coefficient $T$

$$
\begin{aligned}
R(\omega) & =\frac{Z_{1}-Z_{0}-i Y_{0} \omega}{Z_{0}+Z_{1}+i Y_{1} \omega} e^{-i \omega \frac{2 \alpha}{c_{0}}}, \\
T(\omega) & =\frac{2 Z_{2}}{Z_{0}+Z_{1}+i Y_{1} \omega} e^{i \omega\left(\frac{1}{c_{1}}-\frac{1}{c_{0}}\right) \alpha},
\end{aligned}
$$


with

$$
\begin{aligned}
& Z_{0}=\rho_{0} c_{0}, \quad Z_{1}=\rho_{1} c_{1}, \quad Z_{2}=\rho_{0} c_{1}, \\
& Y_{0}=\frac{Z_{0} Z_{1}}{K}-M, \quad Y_{1}=\frac{Z_{0} Z_{1}}{K}+M, \quad Y_{2}=\frac{M}{K} .
\end{aligned}
$$

Note the frequential dependance of $R$ and $T$, induced by $K$ and $M$. If $M=0$ and $K \rightarrow+\infty$, the classical values of $R$ and $T$ are recovered [4]

$$
R=\frac{\rho_{1} c_{1}-\rho_{0} c_{0}}{\rho_{0} c_{0}+\rho_{1} c_{1}}, \quad T=2 \frac{\rho_{0} c_{1}}{\rho_{0} c_{0}+\rho_{1} c_{1}} .
$$

Points 1 and 3 are proposed for one component $\sin \left(\beta_{k} \omega_{c} \xi\right)$ of the initial data (5.3), denoted by $f_{k}(x, t)$. Its Fourier transform in time is

$$
\hat{f}_{k}(\omega)=\frac{\beta_{k} \omega_{c}}{2 \pi}\left(\frac{1}{\omega^{2}-\beta_{k}^{2} \omega_{c}^{2}}\right)\left(e^{-i \frac{2 \pi}{\omega_{c}} \omega}-1\right) .
$$

Reflected and transmitted components are respectively

$$
\hat{g}_{k}(\omega)=R(\omega) \hat{f}_{k}(\omega), \quad \hat{h}_{k}(\omega)=T(\omega) \hat{f}_{k}(\omega) .
$$

The inverse Fourier transform of (B.7) are deduced from classical methods of complex analysis (calculus of residuals). We do not details the steps, and we only give the final results. Setting

$$
\xi_{R}=t+\frac{x}{c_{0}}-\frac{2 \alpha}{c_{0}}, \quad \xi_{T}=t-\frac{x}{c_{1}}+\left(\frac{1}{c_{1}}-\frac{1}{c_{0}}\right) \alpha,
$$

we obtain the reflected component

$$
\begin{aligned}
& \bullet \xi_{R} \leq 0: g_{k}\left(t+\frac{x}{c_{0}}\right)=0 \\
& \bullet 0<\xi_{R} \leq \frac{1}{f_{c}}: \\
& g_{k}\left(t+\frac{x}{c_{0}}\right)=\frac{Z_{1}^{2}-Z_{0}^{2}-Y_{0} Y_{1}\left(\beta_{k} \omega_{c}\right)^{2}}{\left(Z_{0}+Z_{1}\right)^{2}+\left(Y_{1} \beta_{k} \omega_{c}\right)^{2}} \sin \left(\beta_{k} \omega_{c} \xi_{R}\right) \\
& \quad-\frac{2 Z_{0}\left(Z_{1}^{2} / K-M\right)}{\left(Z_{0}+Z_{1}\right)^{2}+\left(Y_{1} \beta_{k} \omega_{c}\right)^{2}} \beta_{k} \omega_{c} \cos \left(\beta_{k} \omega_{c} \xi_{R}\right) \\
& \quad+\frac{2 Z_{0}\left(Z_{1}^{2} / K-M\right)}{\left(Z_{0}+Z_{1}\right)^{2}+\left(Y_{1} \beta_{k} \omega_{c}\right)^{2}} \beta_{k} \omega_{c} e^{-\frac{Z_{0}+Z_{1}}{Y_{1}} \xi_{R}}, \\
& \bullet \xi_{R}>\frac{1}{f_{c}}: \\
& g_{k}\left(t+\frac{x}{c_{0}}\right)=\frac{2 Z_{0}\left(Z_{1}^{2} / K-M\right)}{\left(Z_{0}+Z_{1}\right)^{2}+\left(Y_{1} \beta_{k} \omega_{c}\right)^{2}} \beta_{k} \omega_{c}\left(e^{-\frac{Z_{0}+Z_{1}}{Y_{1}} \xi_{R}}-e^{-\frac{Z_{0}+Z_{1}}{Y_{1}}\left(\xi_{R}-\frac{2 \pi}{\omega_{c}}\right)}\right),
\end{aligned}
$$


and the transmitted component is

$\bullet \xi_{T} \leq 0: h_{k}\left(t-\frac{x}{c_{1}}\right)=0$

$\bullet 0<\xi_{T} \leq \frac{1}{f_{c}}$ :

$h_{k}\left(t-\frac{x}{c_{1}}\right)=\frac{2 Z_{2}\left(Z_{0}+Z_{1}\right)}{\left(Z_{0}+Z_{1}\right)^{2}+\left(Y_{1} \beta_{k} \omega_{c}\right)^{2}} \sin \left(\beta_{k} \omega_{c} \xi_{T}\right)$

$-\frac{2 Z_{2} Y_{1}}{\left(Z_{0}+Z_{1}\right)^{2}+\left(Y_{1} \beta_{k} \omega_{c}\right)^{2}} \beta_{k} \omega_{c} \cos \left(\beta_{k} \omega_{c} \xi_{T}\right)$

$+\frac{2 Z_{2} Y_{1}}{\left(Z_{0}+Z_{1}\right)^{2}+\left(Y_{1} \beta_{k} \omega_{c}\right)^{2}} \beta_{k} \omega_{c} e^{-\frac{Z_{0}+Z_{1}}{Y_{1}} \xi_{T}}$,

- $\xi_{T}>\frac{1}{f_{c}}$ :

$h_{k}\left(t-\frac{x}{c_{1}}\right)=\frac{2 Z_{2} Y_{1}}{\left(Z_{0}+Z_{1}\right)^{2}+\left(Y_{1} \beta_{k} \omega_{c}\right)^{2}} \beta_{k} \omega_{c}\left(e^{-\frac{Z_{0}+Z_{1}}{Y_{1}} \xi_{T}}-e^{-\frac{Z_{0}+Z_{1}}{Y_{1}}\left(\xi_{T}-\frac{2 \pi}{\omega_{c}}\right)}\right)$.

We observe an exponential decay for $\xi_{R}>1 / f_{c}$ in (B.9) and for $\xi_{T}>1 / f_{c}$ in (B.10): unlike the incident wave, reflected and transmitted waves are not spatially bounded. Because the Fourier transform is linear, the components of the total reflected and transmitted field are obtained by summing each component

$$
\begin{aligned}
& g(x, t)=\sum_{k=1}^{q} a_{k} g_{k}(x, t) \\
& h(x, t)=\sum_{k=1}^{q} a_{k} h_{k}(x, t) .
\end{aligned}
$$

Then, the reflected wave $\boldsymbol{U}_{R}(x, t)$ and the transmitted wave $\boldsymbol{U}_{T}(x, t)$ are

$$
\boldsymbol{U}_{R}(x, t)=g(x, t)\left(\begin{array}{c}
\frac{1}{c_{0}} \\
\rho_{0}
\end{array}\right), \quad \boldsymbol{U}_{T}(x, t)=h(x, t)\left(\begin{array}{c}
-\frac{1}{c_{1}} \\
\rho_{1}
\end{array}\right) .
$$

Acknowledgments. Many thanks to Jean-Pierre Sessarego (LMA, Marseille) who supported this research.

\section{REFERENCES}

[1] Y. C. Angel And J. D. AChenbach, Reflexion of ultrasonic waves by an array of microcracks, Review of Progress in Quantitative Nondestructive Evaluation, 4 (1985), pp. 83-89.

[2] J. M. BAIK AND R. B. THOMPSON, Ultrasonic scattering from imperfect interfaces: a quasistatic model, Journal of Nondestructive Evaluation, 4-3 (1985), pp. 177-196.

[3] G. B.van Baren, W. A. Mulder and G.C. Herman, Finite-difference modeling of scalar-wave propagation in cracked media, Geophysics, 66-1 (2001), pp. 267-276.

[4] L. M. Brekhovskikh and O. A. Godin, Acoustics of Layered Media 1-Plane and Quasi-Plane Waves, Springer-Verlag, Berlin Heidelberg, 1990. 
[5] R. T. Coates and M. Schoenberg, Finite-difference modeling of faults and fractures, Geophysics, 69-5 (1998), pp. 1514-1526.

[6] G. Cohen And P. Joly, Construction and analysis of fourth-order finite difference schemes for the elastic wave equation in nonhomogeneous media, SIAM J. Numer. Anal, 33-4 (1996), pp. 1266-1302.

[7] P. P. Delsanto and M. Scalerandi, A spring-model for the determination of the propagation of ultrasonic pulses through imperfect contact interfaces, J. Acoust. Soc. Am., 104-5 (1998), pp. $120-127$.

[8] T. R. Fogarty AND R. J. LEVEQUE, High-resolution finite-volume methods for acoustic waves in periodic and random media, J. Acoust. Soc. Am, 1-106 (1999), pp. 17-28.

[9] T. Fouguet, Raffinement de maillage spatio-temporel local pour les quations de Maxwell, $\mathrm{PhD}$. thesis, University of Paris IX, France (2000).

[10] E. Godlewski and P. A. Raviart, Numerical Approximation of Hyperbolic Systems of Conservation Laws, Springer-Verlag, 1996.

[11] B. Gu, K. NiheI, And L. Myer, Numerical investigation of fracture interface waves, J. Acoust. Soc. Am., 102-1 (1997), pp. 120-127.

[12] B. Gu, K. NiheI, AND L. MYer, Numerical simulation of elastic wave propagation in fractured rock with the boundary integral equation method, J. of Geophys. Res., 101-B7 (1996), pp. 933-943.

[13] G. S. JiAng AND C. W. Shu, Efficient implementation of weighted ENO schemes, J. Comput. Phys., 126 (1996), pp. 202-228.

[14] J. O. Langseth and R. J. LeVeque, A wave-propagation method for three-dimensional hyperbolic conservation laws, J. Comput. Phys, 165 (2000), pp. 126-166.

[15] R. J. LeVeque, Numerical Methods for Conservation Laws, Birkhauser, 1990.

[16] R. J. LeVeque, Wave propagation algorithms for multi-dimensionnal hyperbolic systems, J. Comput. Phys., 13 (1997), pp. 327-353.

[17] Z. LI, The Immersed Interface Method - A Numerical Approach for Partial Differential Equations with Interfaces, PhD. thesis, University of Washington, Seattle, WA, 1994.

[18] Z. Li AND R. J. LeVEque, The Immersed Interface Method for elliptic equations with discontinuous coefficients and singular sources, SIAM J. Num. Anal., 31 (1994), pp. 1019-1044.

[19] B. Lombard, Modélisation numérique de la propagation des ondes acoustiques et élastiques en présence d'interfaces, $\mathrm{PhD}$. thesis, University of Aix-Marseille II, France (2002).

[20] S. Nakagawa, K. Nihei, AND R. MYer, Stop-pass behavior of acoustic waves in a $1 D$ fractured system, J. Acoust. Soc. Am., 107-1 (2000), pp. 40-50.

[21] A. Pilarsky and J. L. Rose, A transverse-wave ultrasonic oblique-incidence technique for interfacial weakness detection in adhesive bonds, J. Acoust. Soc. Am., 63-2 (1988), pp. 300307.

[22] A. Pilarsky, J. L. Rose, and K. Balasubramaniam, The angular and frequency characteristics of reflectivity from a solid layer embedded between two solids with imperfect boundary conditions, J. Acoust. Soc. Am., 87-2 (1990), pp. 532-542.

[23] J. Piraux and B. Lombard, A new interface method for hyperbolic problems with discontinuous coefficients. One-dimensional acoustic example, J. Comput. Phys.,168 (2001), pp. 227248.

[24] M. Punjani and L. J. Bond, A spring-model for the determination of the propagation of ultrasonic pulses through imperfect contact interfaces, Rev. Prog. Quant. Nondestr. Eval., 5-A (1985), pp. 61-71.

[25] S. I. Rokhlin AND Y. J. WAng, Analysis of boundary conditions for elastic wave interaction with an interface between two solids, J. Acoust. Soc. Am., 89-2 (1991), pp. 503-515.

[26] M. Schoenberg, Elastic wave behavior across linear slip interfaces, J. Acoust. Soc. Am., 68-5 (1980), pp. 1516-1521.

[27] J. S. Strikwerda, Finite Difference Schemes and Partial Differential Equations, WadsworthBrooks, New-York, 1989.

[28] H.G. Tattersall, The ultrasonic pulse-echo technique as applied to adhesion testing, J. App. Phys., 6 (1973), pp. 819-832.

[29] A. Wiegmann, The Explicit Jump Immersed Interface Method and Interface Problems for Differential Equations, PhD. thesis, University of Washington, Seattle, WA, 1998.

[30] A. Wiegmann and K. Bube, The Immersed Interface Method for nonlinear differential equations with discontinuous coefficients and singular sources, SIAM J. Numer. Anal, 35 (1998), pp. $177-200$.

[31] A. Wiegmann and K. Bube, The Explicit Jump Immersed Interface Method: finite difference methods for PDE with piecewise smooth solutions, SIAM J. Numer. Anal, 37 (2000), pp. $827-862$. 
[32] C. ZHANG, Immersed Interface Method for Hyperbolic Systems of Partial Differential Equations with Discontinuous Coefficients, PhD. thesis, University of Washington, Seattle, WA, 1996.

[33] C. Zhang And R. J. LeVeque, The Immersed Interface Method for acoustic wave equations with discontinuous coefficients, Wave Motion, 25 (1997), pp. 237-263. 